\title{
Spectrally resolved interferometric observations of $\alpha$ Cephei and physical modeling of fast rotating stars
}

\author{
O. Delaa ${ }^{1,2}$, J. Zorec ${ }^{2}$, A. Domiciano de Souza ${ }^{1}$, D. Mourard ${ }^{1}$, K. Perraut ${ }^{3}$, Ph. Stee ${ }^{1}$, Y. Frémat ${ }^{4}$, J. Monnier ${ }^{7}$, \\ S. Kraus ${ }^{7}$, X. Che ${ }^{7}$, Ph. Bério ${ }^{1}$, D. Bonneau ${ }^{1}$, J. M. Clausse ${ }^{1}$, M. Challouf ${ }^{1}$, R. Ligi ${ }^{1}$, A. Meilland ${ }^{1}$, N. Nardetto ${ }^{1}$,

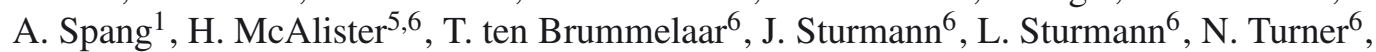 \\ C. Farrington ${ }^{6}$, and P. J. Goldfinger ${ }^{6}$
}

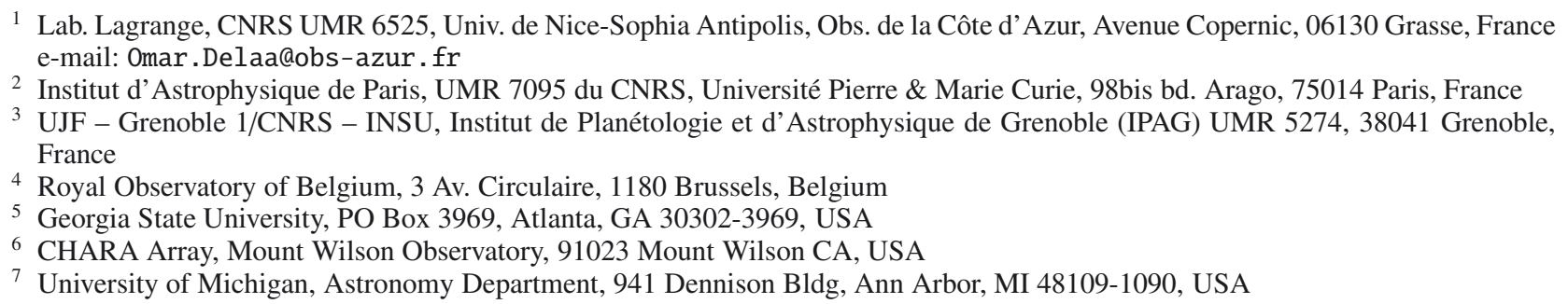

Received 2 November 2012 / Accepted 25 February 2013

\section{ABSTRACT}

\begin{abstract}
Context. When a given observational quantity depends on several stellar physical parameters, it is generally very difficult to obtain observational constraints for each of them individually. Therefore, we studied under which conditions constraints for some individual parameters can be achieved for fast rotators, knowing that their geometry is modified by the rapid rotation which causes a non-uniform surface brightness distribution.

Aims. We aim to study the sensitivity of interferometric observables on the position angle of the rotation axis (PA) of a rapidly rotating star, and whether other physical parameters can influence the determination of PA, and also the influence of the surface differential rotation on the determination of the $\beta$ exponent in the gravity darkening law that enters the interpretation of interferometric observations, using $\alpha$ Cep as a test star.

Methods. We used differential phases obtained from observations carried out in the $\mathrm{H} \alpha$ absorption line of $\alpha$ Cep with the VEGA/CHARA interferometer at high spectral resolution, $R=30000$ to study the kinematics in the atmosphere of the star.

Results. We studied the influence of the gravity darkening effect (GDE) on the determination of the PA of the rotation axis of $\alpha$ Cep and determined its value, $\mathrm{PA}=-157_{-10^{\circ}}^{+17^{\circ}}$. We conclude that the GDE has a weak influence on the dispersed phases. We showed that the surface differential rotation can have a rather strong influence on the determination of the gravity darkening exponent. A new method of determining the inclination angle of the stellar rotational axis is suggested. We conclude that differential phases obtained with spectro-interferometry carried out on the $\mathrm{H} \alpha$ line can in principle lead to an estimate of the stellar inclination angle $i$. However, to determine both $i$ and the differential rotation parameter $\alpha$, lines free from the Stark effect and that have collision-dominated source functions are to be preferred.
\end{abstract}

Key words. techniques: high angular resolution - techniques: interferometric - stars: individual: $\alpha$ Cephei - stars: rotation stars: individual: Aderamin

\section{Introduction}

The centrifugal force of rapid rotators induces a strong deformation of the stellar geometry that has been nicely shown observationally, thanks to long-baseline optical interferometrers that today allow observations at high spatial resolution to be performed (van Belle et al. 2001, 2006; Domiciano de Souza et al. 2003; McAlister et al. 2005; Kervella \& Domiciano de Souza 2006; Monnier et al. 2007; Zhao et al. 2009). Concomitant to the stellar flattening, there is a non-uniform effective temperature distribution, called the gravity darkening effect (GDE; von Zeipel 1924). For stars with conservative rotation laws (rigid rotation is a particular case of a conservative law), whose envelopes are in hydrostatic and radiative equilibrium and where the diffusion approximation to the radiative transfer equation is used, the following relation for the effective temperature distribution as a function of the co-latitude $\theta$ holds:

$T_{\text {eff }}(\theta)=T_{\mathrm{p}}\left[\frac{g_{\mathrm{eff}}(\theta)}{g_{\mathrm{p}}}\right]^{\beta}$,

where $T_{\text {eff }}(\theta)$ and $g_{\text {eff }}(\theta)$ are the co-latitude $\theta$ dependent effective temperature and effective gravity $T_{\mathrm{p}}$ is the polar effective temperature, and $g_{\mathrm{p}}$ is the polar gravity. The canonical value $\beta=0.25$ (i.e., the standard gravity-darkening law von Zeipel 1924 ) is thus valid only in the frame of the above-mentioned physical conditions and approximations. The value $\beta=0.08$ holds for stars with convective envelopes (Lucy 1967; Reiners 2003). Tuominen (1972) also found that $\beta=0.15$ for nonbarotropic differentially rotating stars with masses $M \simeq 2.5 M_{\odot}$. In a recent work, Zorec et al. (2011, and references therein) 
commented that $\beta<0.25$ may reveal a non-conservative rotational law in the stellar surface. Nevertheless, when more detailed expressions for the radiative flux are used and not simply the diffusion approximation, $\beta \lesssim 0.25$ (Claret 2012). Che et al. (2011) suggested $\beta=0.19$ for rapid rotators, and Espinosa Lara $\&$ Rieutord (2012) showed that $\beta$ ranges from 0.25 for slow rotators and decreases for rapid rotators, compatible with observations of Che et al. (2011).

Rotation generates hydrodynamical instabilities that redistribute the angular momentum in the stellar interior. Some of them were taken into account to study their effect on the stellar structure and evolution with rotation (cf. Endal \& Sofia 1979; Pinsonneault et al. 1991; Heger \& Langer 2000; Meynet \& Maeder 2000). These instabilities also induce a turbulent diffusion (Zahn 1992) that, in massive objects, distributes the CNOcycled material from the core to the envelope and changes the stellar atmospheric chemical composition profile (Meynet \& Maeder 2000). Owing to the mass-compensation effect related to the rapid rotation (Sackmann 1970), the total radiative flux produced in the stellar core is lower than that of a parent rotationless star. The combined effect of the mass-compensation effect and the refueling of the stellar core by the meridian circulation contributes to extend the lifespan of stars in the main sequence (cf. Kiziloglu \& Civelek 1996; Talon et al. 1997; Meynet $\&$ Maeder 2000). Stellar winds and the related continuum massloss rate are somewhat modified by the rotation (Lamers \& Cassinelli 1999; Maeder et al. 2007). Maeder et al. (2008) have shown that in massive stars, rapid rotation considerably enlarges the $\mathrm{Fe}$ and $\mathrm{He}$ ionization-related convective zones in their envelope. This convection can then induce a non-conservative, albeit non-shellular, law of differential rotation in the envelope, on which rely both the stellar external geometry and its surface brightness distribution (Zorec et al. 2011).

This account of observational phenomena related with rotation shows that all stellar observables, in particular those we can obtain with interferometry, can be complex functions of several fundamental parameters, some of intrinsic stellar nature (mass, age, chemical composition, amount of angular momentum stored in the star, rotation law, etc.) and others of more geometrical or extrinsic character (inclination angle, position in the sky, etc.). The stellar geometry and the wavelength-dependent surface brightness distribution are both measurable with interferometric techniques. We can then ask whether by using observables, some of the quoted independent parameters can be tested individually, or whether they should all be determined and analyzed at once.

At a first glance, we may expect that due to their extrinsic nature, the position angle of the rotation axis and the stellar inclination can be determined independently of the stellar parameters of intrinsic character, even though the determination of the inclination angle relies on the surface brightness asymmetries, which in turn are controlled by intrinsic stellar quantities.

The aim of the present paper is the following: thanks to a spectral resolution that can reach 30000 , the VEGA/CHARA instrument (Mourard et al. 2009, 2010) offers a great possibility to perform precise measurements of differential phases of fringes to study the prevailing kinematics in the stellar atmospheres. By taking advantage of the link that exists between the phase of fringes and the position of the photo-center in a partially resolved star, we can carry out spectro-interferometric studies and provide measurements of the position angle (PA) of stars. A similar approach was recently used to measure the PA of the rotation axis of the fast rotators Fomalhaut (Le Bouquin et al. 2009) and Achernar (Domiciano de Souza et al. 2012a). In addition, this work differs from previous studies of the PA (van Belle et al. 2006; Zhao et al. 2009), because we use information on the stellar atmosphere from the observed spectral lines. The method is tested for $\alpha$ Cep using the $\mathrm{H} \alpha$ absorption line.

In principle, the combination of the high spectral and spatial resolution should enable us to extract information from the surface radial iso-velocity curves, whose properties reflect the kinematics in the stellar atmosphere. The apparent aspect of the radial iso-velocity curves depends, however, on the inclination angle $i$ of the rotation axis, and on the degree of the surface differential rotation. It is then worthwhile to discuss whether we can estimate the inclination angle $i$ and the degree of differential rotation independently of the intrinsic stellar parameters.

The present paper is organized as follows: in Sect. 2 we summarize previous studies of $\alpha$ Cep. In Sect. 3, we present our interferometric observations and reduction technique. In Sect. 4, we introduce our method for deriving the position angle PA of the stellar rotation axis. In this section, we also introduce the model used to interpret the interferometric measurements and present the results obtained with our best model. In Sect. 5 we study the effects on the von Zeipel coefficient $\beta$ determination that depend on the law of surface differential rotation and analyze the feasibility of the inclination angle $i$ determination. The conclusions are summarized in Sect. 6.

\section{2. $\alpha$ Cephei}

$\alpha$ Cephei (Alderamin, HD 203280 , HR 8162, $V=2.46, d=$ $14 \mathrm{pc}$ ) is the brightest star in the constellation of Cepheus. Originally classified as A2n (Douglas 1926), the spectral type of $\alpha$ Cephei has been re-estimated in Johnson \& Morgan (1953), who classified it as A7IV-V. Gray et al. (2003) classified the star as A8V. Like Altair, $\alpha$ Cep has a chromosphere (Walter et al. 1995; Simon \& Landsman 1997; Simon et al. 2002). According to various authors, the $V \sin i$ of $\alpha$ Cep ranges from $180 \mathrm{~km} \mathrm{~s}^{-1}$ to $245 \mathrm{~km} \mathrm{~s}^{-1}$ (Bernacca \& Perinotto 1973a,b,c; Uesugi \& Fukuda 1970; Royer et al. 2007; Abt \& Morrell 1995).

Up to now, few interferometric observations have been carried out on $\alpha$ Cep. van Belle et al. (2006) used the Georgia State University CHARA array to observe this star in the infrared and estimated its oblateness, the rotational velocity, and its gravity darkening. Their data showed a non-circular projected disk brightness distribution from which they deduced the equatorial and polar diameter of $\alpha$ Cep using two different models. The first one, purely geometrical, represents an elliptical star whose brightness distribution is uniform. From this model, they found a polar diameter of $1.355 \pm 0.080$ mas, an equatorial diameter of $1.625 \pm 0.056$ mas and a position angle of the rotation axis of $3^{\circ} \pm 14^{\circ}$ (or $-177^{\circ}$, since there can be an indetermination of $180^{\circ}$ ) with a reduced $\chi^{2}$ of 1.08 . The second, and more physical model, takes into account the shape of the photosphere under conditions of hydrostatic equilibrium, uniform rotation, Roche's approximation for the total potential at the surface, the limb darkening that responds to the geometrical deformation of the star, and the surface gravity darkening. The parameters derived from their best model are summarized in Table 1 . Their estimate of the $\beta$ exponent in the gravitational darkening law gives $\beta=0.084_{-0.049}^{+0.026}$, which is consistent with $\beta=0.08$ expected for a star whose envelope is entirely convective (Lucy 1967; Reiners 2003).

Taking advantage of a better $(u, v)$-plane coverage than in van Belle et al. (2006), Zhao et al. (2009) used the Michigan Infra-Red Combiner (MIRC) at the CHARA Array to produce a model for $\alpha$ Cep as fast rotator. These authors calculated models of brightness distribution in fast rotating stars according to 
O. Delaa et al.: Spectrally resolved interferometric observations of $\alpha$ Cep and physical modeling of fast rotating stars

Table 1. Parameters from best models obtained by van Belle et al. (2006) and Zhao et al. (2009).

\begin{tabular}{lcc}
\hline \hline Model parameters & Van Belle et al. (2006) & Zhao et al. (2009) \\
\hline$i$ & $88.2^{\circ+1.8^{\circ}}$ & $55.70^{\circ} \pm 6.23^{\circ}$ \\
PA & $-163^{\circ}+3.2^{\circ}$ & $-178.84^{\circ} \pm 4.28^{\circ}$ \\
$T_{\mathrm{p}}$ & $8440_{-700}^{+430} \mathrm{~K}$ & $8588 \pm 300 \mathrm{~K}$ \\
$R_{\mathrm{p}} / R_{\odot}$ & $2.175 \pm 0.046$ & $2.162 \pm 0.036$ \\
$T_{\text {eq }}$ & $7600 \mathrm{~K}$ & $6574 \pm 200 \mathrm{~K}$ \\
$R_{\mathrm{eq}} / R_{\odot}$ & $2.823 \pm 0.097$ & $2.740 \pm 0.044$ \\
$\Omega / \Omega_{\mathrm{c}}$ & $0.8287_{-0.0232}^{+0.0482}$ & $0.941 \pm 0.020$ \\
$\beta$ & $0.084_{-0.049}^{+0.026}$ & $0.216 \pm 0.021$ \\
$V \sin i$ & $283 \pm 19 \mathrm{~km} \mathrm{~s}^{-1}$ & $225 \mathrm{~km} \mathrm{~s}^{-1}$ \\
$\chi_{\text {red }}^{2}$ & 2.16 & 1.18 \\
\hline
\end{tabular}

prescriptions given by Aufdenberg et al. (2006, and references therein), which they fitted to the interferometric observations. In these models the authors assumed solid-body rotation, adopted a Roche potential to characterize the stellar surface, and used the von Zeipel gravity darkening law (von Zeipel 1924) to determine the latitude-dependent effective temperature profile. Hence, for $\alpha$ Cep they deduced the inclination angle $i$, the position angle PA of the rotation axis, the polar $R_{\text {pol }}$ and equatorial $R_{\text {eq }}$ radii, the polar effective temperature $T_{\mathrm{p}}$, and equatorial effective temperature $T_{\text {eq }}$, the angular velocity ratio $\omega=\Omega / \Omega_{\mathrm{c}}$, and the $\beta$ exponent. These values are summarized in Table 1 .

The exponent $\beta=0.216 \pm 0.021$ found by Zhao et al. (2009) suggests that the envelope of $\alpha$ Cep is probably not convective as found by van Belle et al. (2006). Because their $\beta$ exponent is lower than 0.25 , at least three possibilities exist: 1 ) the stellar envelope is mostly radiative but interspersed with convective regions, which in the equatorial region case perhaps induced by the GDE where the effective temperature is low enough; 2) the surface rotation reflects a deeper non-conservative rotation law in the envelope; or 3) the diffusion approximation used to derive the gravity darkening law is not sufficient, and more detailed expressions for the emerging radiative flux must be taken into account (Claret 2012).

\section{Observations and data reduction}

\subsection{Observations}

The VEGA instrument (Mourard et al. 2009) at the CHARA Array (ten Brummelaar et al. 2005), located on the Mount Wilson Observatory (LA, California, USA), operates in the visible domain and benefits from a spectrograph and a polarimeter. The spectrograph is designed to sample in the visible band from 0.45 to $0.85 \mu \mathrm{m}$ and is equipped with two photon-counting detectors that observe in two different spectral bands (the detector that observes at the shortest wavelength is denoted "blue", the other is called "red"). Interferometric observations of $\alpha$ Cep with the VEGA/CHARA instrument were carried out in July 2008 and October 2010 using the high spectral resolution mode $(R=$ $30000)$. All observations were made with two telescopes only. Observations were conducted with the red and blue detectors centered at $656 \mathrm{~nm}(\mathrm{H} \alpha$ line) and $632 \mathrm{~nm}$, respectively. In this spectral resolution mode, the $\mathrm{H} \alpha$ line covers almost the entire surface of the red detector, which allows us to make a precise measurement of the differential phase. Because we based our study on a differential analysis, we did not need to observe

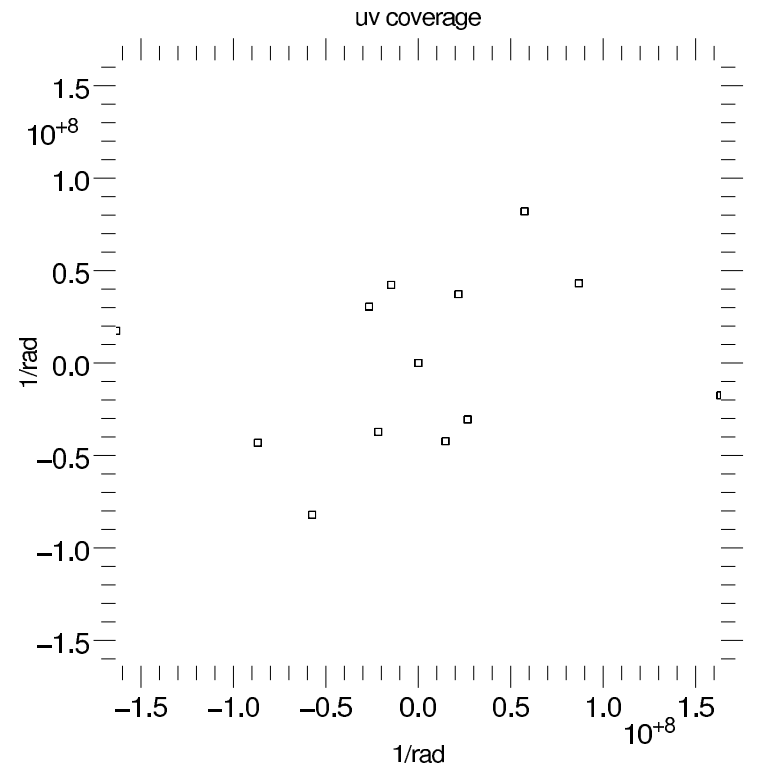

Fig. 1. $(u, v)$-plane coverage by our observations (u is in abscissas).

Table 2. Log of observations with VEGA/CHARA.

\begin{tabular}{|c|c|c|c|c|c|}
\hline \multicolumn{2}{|c|}{ Date (UTC) } & \multirow[t]{2}{*}{ Telescopes } & \multicolumn{2}{|c|}{ Projected baseline } & \multirow{2}{*}{$\begin{array}{c}r_{0} \\
(\mathrm{~cm})\end{array}$} \\
\hline & & & & & \\
\hline $28 / 0$ & $03 \mathrm{~h} 44$ & & 284 & +29 . & 8 \\
\hline $28 /($ & 09h & & 9.3 & & 13 \\
\hline $28 / 07 / 2008$ & $11 \mathrm{~h} 48$ & S & 26.4 & $-42.1^{\circ}$ & 8 \\
\hline $13 / 10 / 2010$ & 03h02 & E1l & 63.6 & $-116.4^{\circ}$ & 8 \\
\hline $13 / 10 / 2010$ & 03h52 & E1 & 65.8 & $-125.7^{\circ}$ & 8 \\
\hline $13 / 10 / 2010$ & 04h59 & $\mathrm{E} 1 \mathrm{E} 2_{c}$ & 65.7 & $-145.0^{\circ}$ & 7 \\
\hline
\end{tabular}

Notes. $r_{0}$ is the Fried turbulence parameter; sub-indices $a, b$ and $c$ identify the observing dates.

calibrators. The log of the observations is given in Table 2 and the $(u, v)$-plane coverage during these observation campaigns is shown in Fig. 1.

\subsection{Data reduction}

The data were reduced with the software developed in the VEGA group and described by Mourard et al. (2009). This software offers two data reduction modes, the first one is the spectral density analysis and the second one the cross-spectral analysis scheme. In the spectral density mode, we only obtain the squared visibility averaged over a spectral band centered at a given wavelength $\lambda_{1}$. With the cross-spectral analysis mode, we obtain a visibility modulus $V(\lambda)$ and a differential phase $\phi_{\text {diff }}$ within a narrow channel of a few angstroms, which is step-by-step translated over a large reference spectral band. In each step, we measure the product $V\left(\lambda_{1}\right) \times V\left(\lambda_{2}\right)$ and $\phi_{\text {diff }}\left(\lambda_{1}\right)$. In this study, we are only interested by the quantity $\phi_{\text {diff }}\left(\lambda_{1}\right)$. The spectral calibration was performed with known spectral lines of the ThAr spectral lamp. A polynomial fit was made to determine the dispersion law. The residual of the fit is typically two times lower than the spectral resolution.

Figure 2 presents the differential phases obtained after the data processing. In this figure we also superimposed (red solid line) the best-fit model. As explained in Sect. 4.4, the differential phases in the wings of the $\mathrm{H}_{\alpha}$ line (outside $6555 \AA$ to $6569 \AA$ ) can be considered to be equal to zero. We used the measurements 

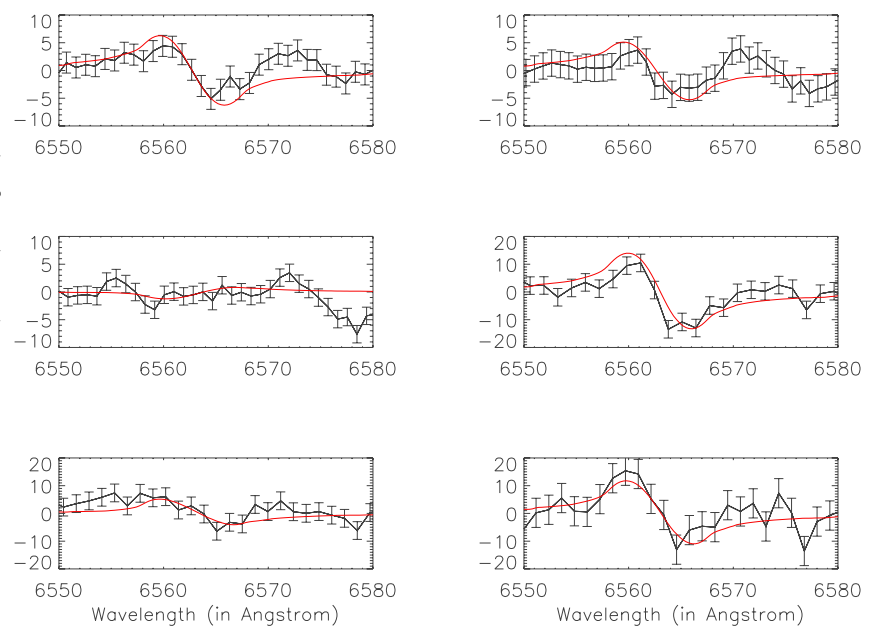

Fig. 2. Differential phases obtained after data processing. This figure also shows the best-fit (red line) models calculated as a function of the position angle of the rotation axis $\left(\mathrm{PA}=-157_{-10^{\circ}}^{+10^{\circ}}\right.$ ) obtained as explained in Sect. 4.4.

in this "wings" region to estimate the variance of the phase and decided to attribute this variance to the individual phase error. We then applied a fit of the model in the S-shaped region, where a signal is expected, i.e. from $6555 \AA$ to $6569 \AA$.

\section{Determination of the position angle}

\subsection{Principles}

Interferometric observables were modeled from a computed brightness distribution (see Sect. 4.2) using the Zernike-van Cittert's theorem. This theorem relates the complex visibility $\mathscr{V}_{\delta \lambda}$ with a stellar surface brightness distribution emitted in a given spectral channel of bandwidth $\delta \lambda$ :

$\mathscr{V}_{\delta \lambda}(u, v)=\frac{\int_{-\infty}^{\infty} \int_{-\infty}^{\infty} I_{\delta \lambda}(\alpha, \beta) \exp [-2 \pi i(\alpha u+\beta u)] \mathrm{d} \alpha \mathrm{d} \beta}{\int_{-\infty}^{\infty} \int_{-\infty}^{\infty} I_{\delta \lambda}(\alpha, \beta) \mathrm{d} \alpha \mathrm{d} \beta}$,

where $I_{\delta \lambda}(\alpha, \beta)$ is the intensity of the emitted energy by the source toward the observer caught in the spectral channel of bandwidth $\delta \lambda$ and centered on the wavelength $\lambda ;(\alpha, \beta)$ are the angular coordinates of an emitting point in the source projected onto the sky, while $(u, v)$ are the Fourier conjugate spatial frequencies related to the baseline coordinates and measured in the observing plane. The modulus of the complex visibility, $V_{\delta \lambda}=\left|\mathscr{V}_{\delta \lambda}(u, v)\right|$, measures the contrast of the interferometric fringes, while its argument determines the phase of the fringes, $\phi_{\delta \lambda}=\arg \left[\mathscr{V}_{\delta \lambda}(u, v)\right]$. For an unresolved source, $\phi_{\delta \lambda}$ is directly related to the position of the photo-center of the brightness distribution at a given wavelength $\lambda$. Unfortunately, the atmospheric turbulence disrupts the phase of fringes when interferometric observations are performed with only two telescopes. To overcome this difficulty, we used the differential phase $\phi_{\text {diff }}$, defined as the difference between the phase measured in two spectral channels centered on the wavelengths $\lambda_{1}$ and $\lambda_{2}$ :

$\phi_{\text {diff }}=\phi_{\lambda_{2}}-\phi_{\lambda_{1}}$.

The quantity $\phi_{\text {diff }}$ enables us to quantify the displacement of photo-centers at different wavelengths $\lambda$ of an unresolved source free from perturbations from the atmospheric turbulence. The spatial resolution reached with the S1S2 and E1E2 baselines used in this study are 5.5 and 2.7 mas, respectiveley, at $0.656 \mu \mathrm{m}$.
Hence, $\alpha$ Cep remained unresolved (see Table 1). The crossspectrum method described in Berio et al. (1999) and Tatulli et al. (2007) used in the VEGA/CHARA data processing aims at measuring the differential phases $\phi_{\text {diff }}(\lambda)$ and the differential visibility $V_{\text {diff }}(\lambda)$ between two different spectral channels centered on the wavelengths $\lambda_{1}$ and $\lambda_{2}$.

\subsection{Models of stellar surface brightness distribution}

To calculate $\phi_{\text {diff }}$ and $V_{\text {diff }}(\lambda)$ we used the model described in Domiciano de Souza et al. (2002) and Domiciano de Souza et al. (2012b), which can produce the intensity maps in the $\mathrm{H} \alpha$ line needed in this work. This model is based on the following assumptions: a) stars have rigid rotation; b) Roche-approximation is used to describe the stellar shape modified by the rigid rotation; c) at each point of the observed stellar hemisphere, the atmosphere is approximated by a plane-parallel model for the local effective temperature $T_{\text {eff }}(\theta)$ and gravity $g(\theta)$.

A modified version of the BRUCE/KYLIE calculation code (Townsend 1997) was used to divide the apparent hemisphere into elementary surface elements (blocks), each characterized by a given set of local physical parameters. The plane-parallel stellar atmosphere models were computed using the Kurucz stellar atmosphere models with solar abundances (Kurucz 1979). The SYNSPEC code (Hubeny \& Lanz 1995) with chemical compositions compatible with metallicity $Z=0.02$ was employed to calculate the local emergent specific radiation intensities directed toward the observer $I_{\delta \lambda}(\alpha, \beta)$ which determine the monochromatic intensity maps of the apparent photosphere in the wavelengths of the instrumental transmitting channels. The models depend on five intrinsic stellar parameters: polar radius $R_{\mathrm{p}}$, the polar temperature $T_{\mathrm{p}}$, the polar gravity $g_{\mathrm{p}}$, the stellar rotational flattening $D=R_{\mathrm{e}} / R_{\mathrm{p}}$, and the gravity darkening exponent $\beta$. In addition, there are two extrinsic parameters: the stellar inclination angle $i$ and the position angle PA of the stellar rotation axis.

\subsection{Method}

We adopted a Cartesian reference system in the sky centered on the star, so that the $x$-axis lies along the equator of the star and the $y$-axis contains the plane of the stellar rotation axis. In the upper panel of Fig. 3, we show the computed displacement of the photo-center of the $\mathrm{H}_{\alpha}$ line as observed along the equatorial axis of $\alpha$ Cep. The corresponding differential phases $\phi_{\text {diff }}(\lambda)$ are shown in the lower panel of Fig. 3. Phases are calculated for a sixty-meter baseline parallel to the direction of the stellar equatorial axis. When the orientation of the baseline projected onto the sky changes with respect to the $x$-axis, we obtain different plots of $\phi_{\text {diff }}(\lambda)$, as illustrated in Fig. 4, where the line-styles change according to the orientation of the baseline. $\phi_{\text {diff }}(\lambda)$ is well spread for a baseline perpendicular to the rotational axis of the star (Mourard 1990), whereas for a baseline parallel to the rotational axis of the star, $\phi_{\text {diff }}(\lambda)$ is close to zero. So, to determine the position angle PA of the stellar rotation axis of $\alpha$ Cep, we carried out spectro-interferometric observations at different orientations of the baseline and took measurements of the respective variation of phases $\phi_{\text {diff }}(\lambda)$. Thus, for a given observed distribution $\phi_{\text {diff }}(\lambda)$ as a function of $\lambda$, we can estimate the PA by searching the best fit of the observed differential phases with calculated ones for different position angles. 
O. Delaa et al.: Spectrally resolved interferometric observations of $\alpha$ Cep and physical modeling of fast rotating stars
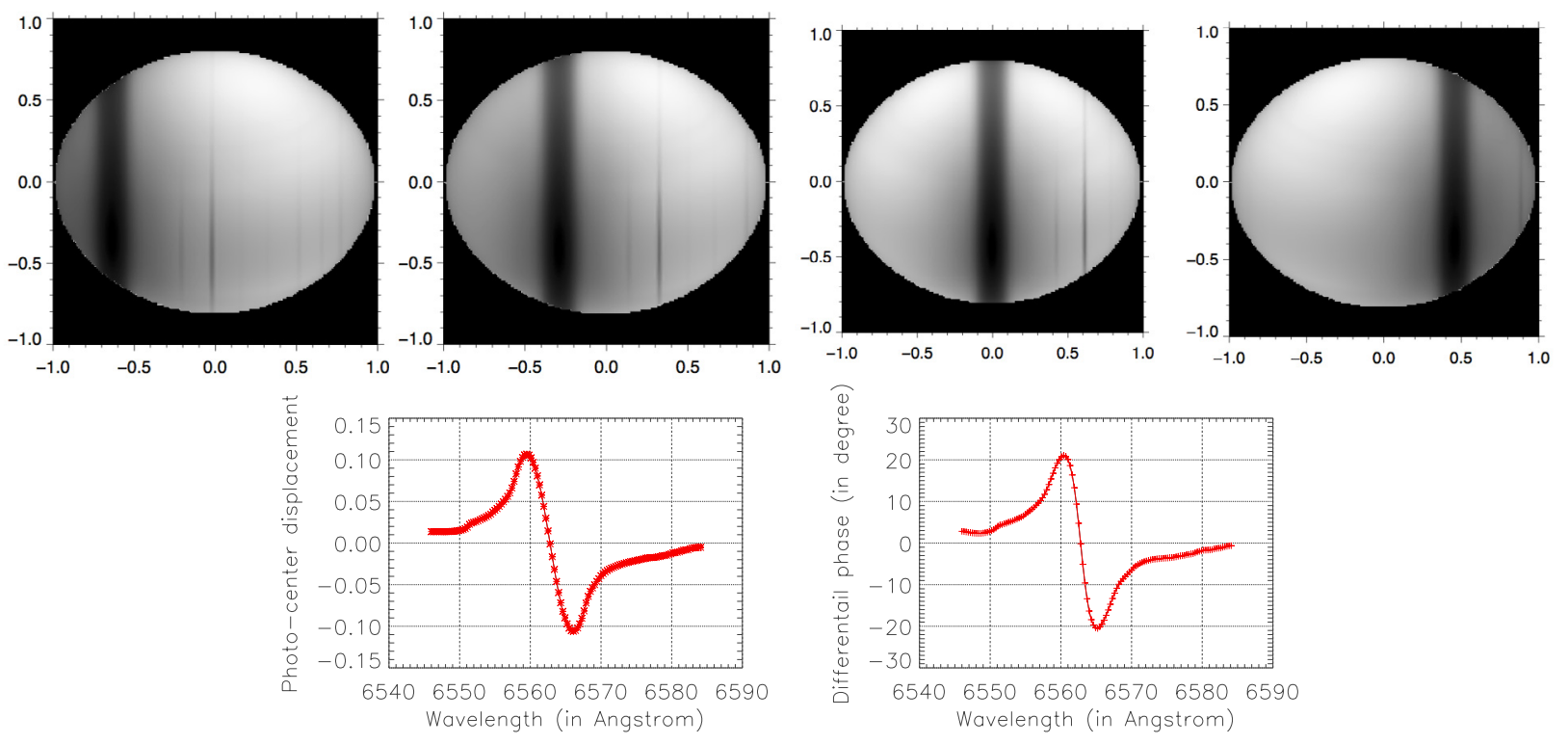

Fig. 3. Top: intensity maps calculated for selected wavelengths inside the $\mathrm{H} \alpha$ line (from left to right): $6559.5 \AA$, $6561.3 \AA$, $6562.8 \AA$, and $6565.2 \AA$. Ordinates and abscissas are given in units of the equatorial radius $R_{\text {eq. }}$. The inclination angle used is $i=55^{\circ}$. Bottom: photo-center displacements along the $y$-axis (left side); differential phase associated with the photo-center shift (right side). All quantities are computed for a sixty-meter baseline parallel to the equatorial axis ( $x$-axis). The photo-center displacements are given in units of the angular stellar radius.
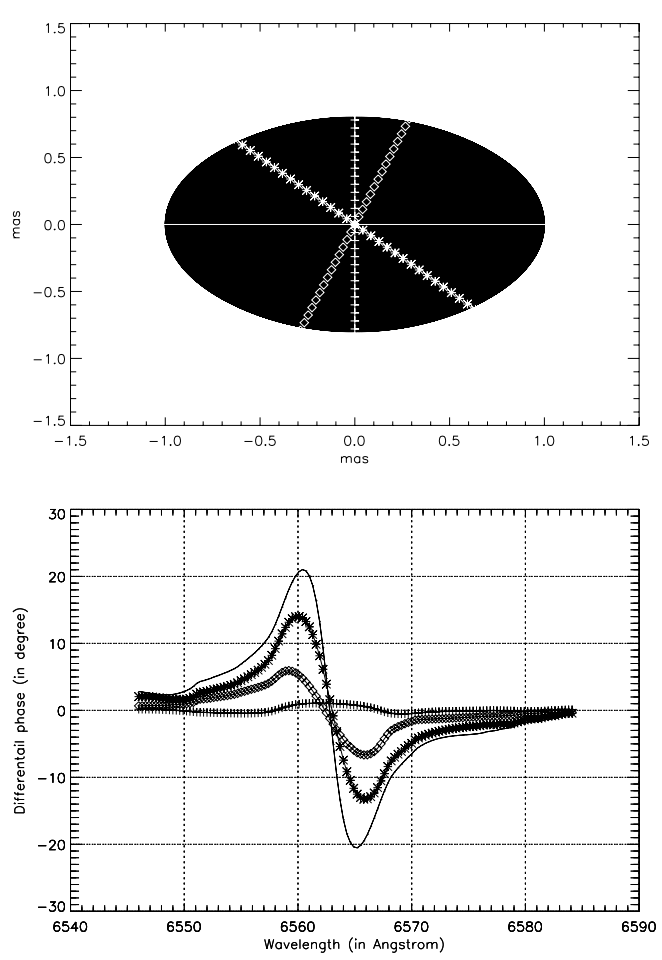

Fig. 4. Differential phases $\phi_{\text {diff }}(\lambda)$ in the $\mathrm{H}_{\alpha}$ line for different baseline orientations. Top: stellar disk deformed by the fast rotation and projected onto the sky where we have represented the baseline orientations: $0^{\circ}$ (cross), $45^{\circ}$ (star), $90^{\circ}$ (solid line) and $160^{\circ}$ (diamond). Bottom: evolution of $\phi_{\text {diff }}(\lambda)$ according to the baseline orientations represented on top and computed for a sixty meter-baseline. In this example we assumed that the position angle of the stellar rotation axis is equal to zero.

\subsection{Model fitting}

The position angle PA of the rotation axis is an extrinsic parameter whose determination can be assumed independent of

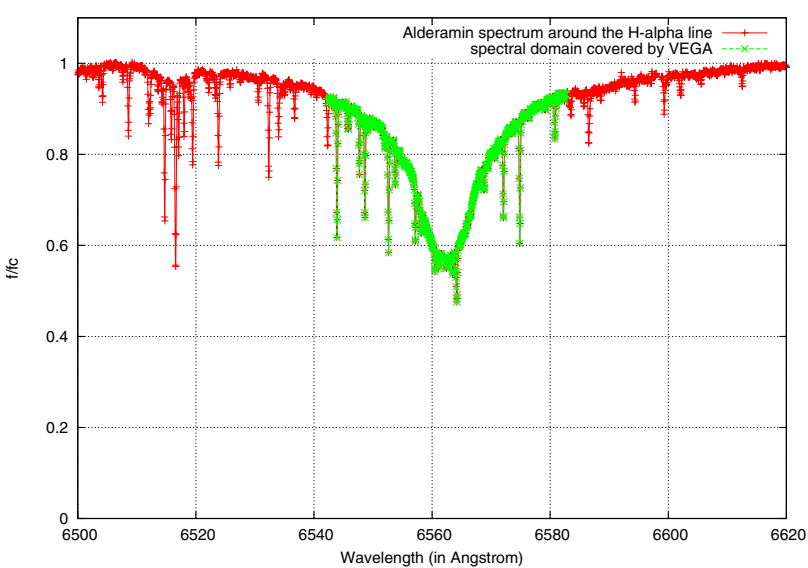

Fig. 5. Spectrum of alpha Cep obtained in the high spectral resolution mode of VEGA/CHARA (green) and ith the ELODIE spectrograph (red).

the choice of the intrinsic stellar parameters. The results obtained will justify this assumption a posteriori. So, to calculate the brightness distribution, we adopted the stellar quantities $R_{\mathrm{p}}$, $T_{\mathrm{p}}, g_{\mathrm{p}}, D$, and $i$ obtained by Zhao et al. (2009, see Table 1$)$ because of their excellent $(u, v)$-plane coverage. Then, we calculated monochromatic intensity maps for wavelengths inside the $\mathrm{H} \alpha$ line as detailed in Sect. 4.2, and for a series of angle PA and $\beta$ parameters. The corresponding differential phases $\phi_{\text {diff }}(\lambda)$ were estimated by calculating the FT of the monochromatic intensity maps.

It is important to note that in the high spectral resolution mode, the red detector of the VEGA/CHARA instrument covers only the spectral interval $\lambda \lambda 6542.49-6582.68 \AA$ of the observed $\mathrm{H}_{\alpha}$ line of $\alpha \mathrm{Cep}$, as shown in Fig. 5. Because of the lack of measurements in the continuum spectrum around the line, we calibrated the differential phases by assuming that both line wings in the uncovered spectral ranges are symmetric. To test this hypothesis, we computed images of the apparent stellar 

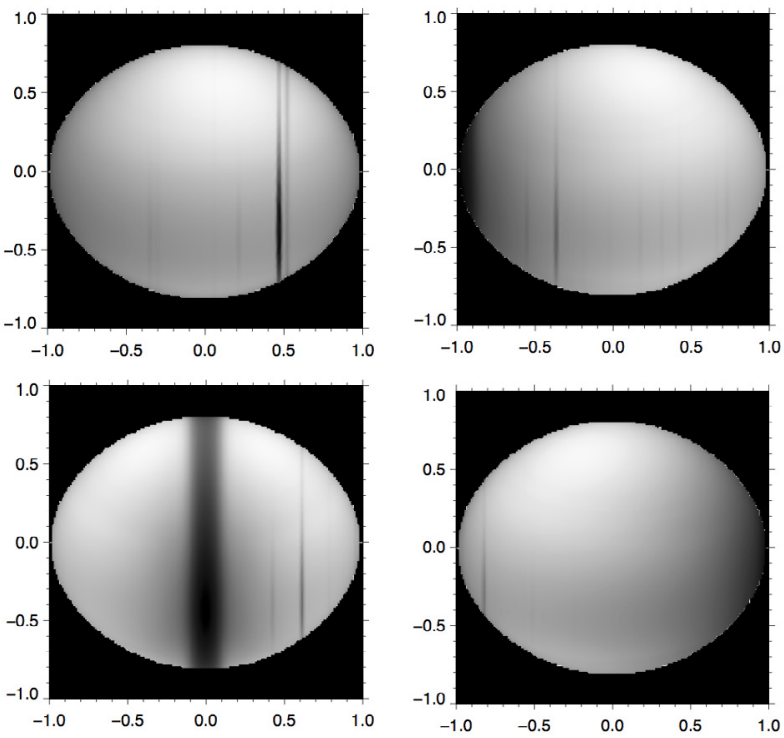

Fig. 6. Surface brightness distribution in $\alpha$ Cep at four different wavelengths: $6548.7 \AA$ (top, left), $6557.7 \AA$ (top, right), $\lambda 6562.8 \AA$ (bottom, left), and $\lambda 6568.5 \AA$, (bottom, right). Ordinates and abscissas are given in units of the equatorial radius $R_{\text {eq }}$. The inclination angle used is $i=55^{\circ}$.

Table 3. Differential phases as a function of different wavelengths inside the $\mathrm{H}_{\alpha}$ line for the simulated intensity maps of Fig. 6 .

\begin{tabular}{lc}
\hline \hline$\lambda(\AA)$ & $\phi_{\text {diff }}(\lambda)$ \\
\hline 6548.7 & $3^{\circ}$ \\
6557.7 & $4^{\circ}$ \\
6562.8 & $0^{\circ}$ \\
6568.5 & $4^{\circ}$ \\
\hline
\end{tabular}

hemisphere and the respective differential phases at four different wavelengths: $\lambda$ 6548.7, 6557.7, 6562.8, and 6568.5. A perfectly symmetric distribution of the stellar brightness in the given wavelengths should lead the corresponding photocenters to lie in the center of the disk and the respective differential phases to be zero. The images of the brightness distribution over the stellar disk at the chosen wavelength are shown in Fig. 6. The values of the differential phase for each image are summarized Table 3 . Because the expected uncertainty on the estimate of differential phases is roughly $3^{\circ}$, we conclude that in the wavelength-intervals $\lambda \lambda 6542.49-6557.7 \AA$ and $\lambda \lambda 6568.5-6582.68 \AA$ our hypothesis of wing-symmetry is a reasonable first approximation.

According to the results shown in Table 3, we can assume that the differential phase in the wings of the $\mathrm{H} \alpha$ line is equal to zero, because we were dealing with the continuum spectrum proper. The differential phases in these spectral ranges were fitted with a polynomial and then used to divide the observed differential phases to obtain the sought calibration.

To determine PA, a grid of models parameterized according to the exponent $\beta$, and the position angle of the rotation axis of $\alpha$ Cep was produced. The $\beta$ exponents used range from 0.08 to 0.25 by steps of 0.01 , while the angle PA ranged from $-130^{\circ}$ to $-180^{\circ}$ by steps of $1^{\circ}$. This interval of PA was chosen according to previous indications on the PA value inferred in van Belle et al. (2006) and Zhao et al. (2009). Each model provides thus the observable $\phi_{\text {diff }}(\lambda)$, which is compared to the data and the fit controlled through a $\chi^{2}$ test. In Fig. 2 are

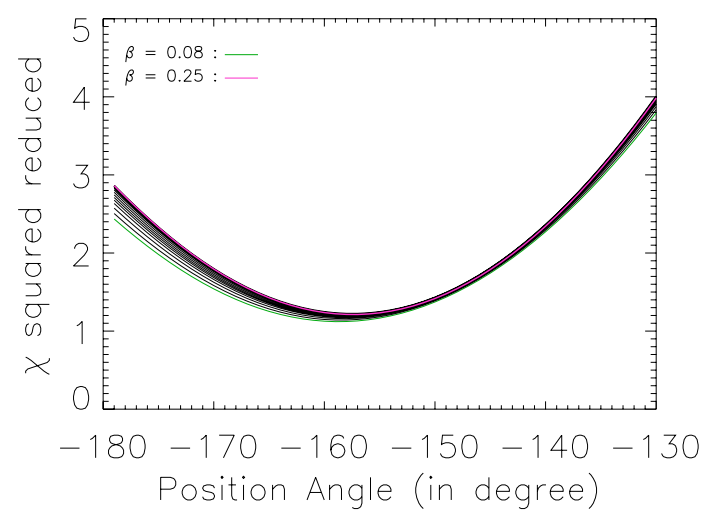

Fig. 7. $\chi_{\text {red }}^{2}$ as a function of the PA of the rotation axis of $\alpha$ Cep for different von Zeipel exponents ranging from $\beta=0.08$ to $\beta=0.25$.

shown the respective best fits obtained (red lines) to the observed differential phases. We computed the reduced $\chi^{2}$ residuals (hereafter $\left.\chi_{\text {red }}\right)$ and plotted as a function of the PA angle, as shown in Fig. 7, where each curve corresponds to a different gravity darkening exponent. We note that the $\chi_{\text {red }}$ curves are calculated for $\lambda 6560 \AA$ and each of them is an average over the six better fits obtained from the respective observed differential phases.

From the $\chi_{\text {red }}$ curves in Fig. 7 we can see that if the PA determination corresponds to the minimum value $\left[\chi^{2}(\beta)_{\text {red }}\right]_{\min }$, roughly the same angle PA will be obtained whatever the $\beta$ exponent. Because each $\beta$ value produces a different effective temperature contrast over the observed stellar hemisphere, with the consequent contrast of the emitted radiative fluxes, which mimic the effect of different sets of intrinsic stellar parameters, we conclude that the observed differential phases $\phi_{\text {diff }}(\lambda)$ against the position angle reflect the external stellar geometry quite independently of any explicit intrinsic physical parameter.

To avoid inconveniences introduced by possible correlations among data points, a reliable approach to the uncertainty affecting the estimate of the position angle PA can be inferred by using the random re-sampling of data or "bootstrap simulation" (Efron \& Tibshirani 1986). We thus randomly re-sampled $B=700$ times the six data sets of differential phases shown in Fig. 8 in the wavelength interval used to fit the model-differential phases. This leads to $B$ independent determinations of PA values shown in Fig. 8a against the bootstrap replication number. The position angles of Fig. 8a were calculated for the von Zeipel coefficient $\beta=0.19$ that was suggested in Che et al. (2011) to analyze interferometric data. The position angles PA were then gathered into a histogram of frequencies where the class-step (bin-width) $\triangle \mathrm{PA}=2^{\circ}$ was chosen following the bin-width optimization method in Shimazaki \& Shinomoto (2007). The histogram is shown in Fig. 8b, where we also show a smoothed version of the histogram obtained using the Gaussian-kernelestimator method (Bowman \& Azzalini 1997) (dashed curve). We can readily see that the estimate of PA with the highest probability is $\mathrm{PA}=-157^{\circ}$, while more than $99 \%$ of estimates are in the interval from $\mathrm{PA}=-167^{\circ}$ to $\mathrm{PA}=-140^{\circ}\left(\mathrm{PA}=-157_{-10^{\circ}}^{\circ}\right)$. The whole fitting procedure was also repeated for $\beta=0.08$ and $\beta=0.25$. While the confidence interval remains in all cases the same, for $\beta=0.08$ the maximum is slightly shifted to more negative values of PA and slightly to more positive PA for $\beta=0.25$ than with $\beta=0.19$, but the whole displacement implies $\Delta \mathrm{PA} \simeq 2^{\circ}$. The low value of $\Delta \beta$ demonstrates the high independence of the PA-angle determination on the chosen value of $\beta$. 
O. Delaa et al.: Spectrally resolved interferometric observations of $\alpha$ Cep and physical modeling of fast rotating stars
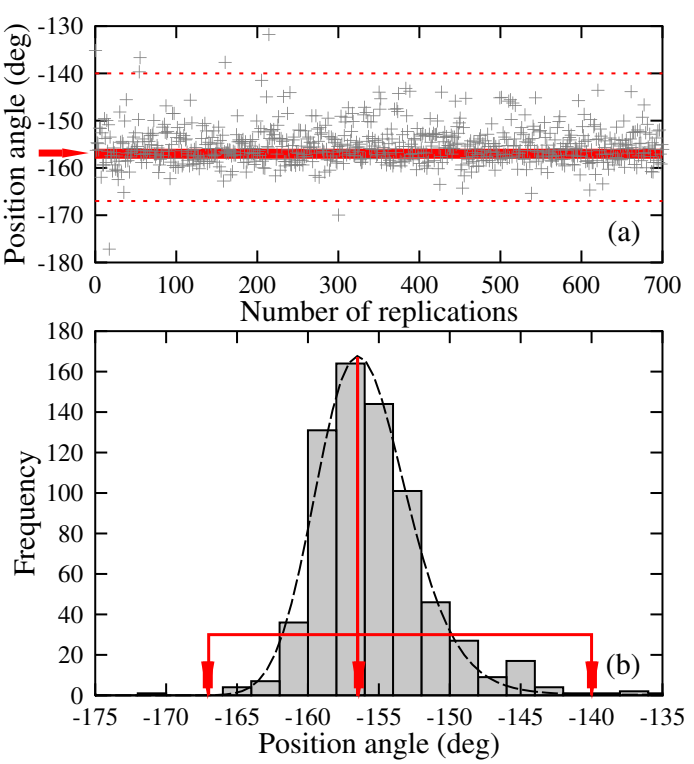

Fig. 8. a) Simulated position angle PA against the number of "bootstrap" replications when $\beta=0.19$. The colored line indicates the position angle PA of maximum probability. b) Frequency distribution of the simulated position angles PA (histogram and smoothed distribution). In the figure are also indicated the position angle PA of maximum probability and the $99 \%$ confidence interval.

The contrast of the brightness distribution over the stellar surface was determined simultaneously: a) the rotation law, characterized in Sect. 5.1 by the parameter $\alpha$; b) the value of the $\beta$ exponent; and c) the equatorial angular velocity $\Omega_{\mathrm{eq}}$. It can be expected that some degeneracy of $\alpha, \Omega_{\mathrm{eq}}$, and $\beta$ may appear when they are determined together from the observed stellar surface brightness distributions. Accordingly, there may also appear some indetermination of the inclination angle, since the $V \sin i$, a key quantity for estimating $i$, depends on $V_{\text {eq }}$, which in turn is determined by $\Omega_{\mathrm{eq}}$ and the true stellar equatorial radius $R_{\mathrm{eq}}$. In this section we have taken a wide range of possible values of $\beta$, which can be thought of as blurring uncertainties on the surface brightness distribution carried by $\alpha$ and $\Omega_{\mathrm{eq}}$. We can conclude that at least the PA angle can be safely determined even though other intrinsic stellar quantities are uncertain.

\section{Inclination angle and surface differential rotation}

In this section we explore whether the inclination angle $i$ can be estimated with interferometric observations and what effects are induced by the surface differential rotation that may hamper the angle $i$-determination.

Compared to the rigid rotation where the angular velocity $\Omega(\theta)$ is constant over the entire stellar surface, the differential rotation introduces a supplementary $\theta$-dependent centrifugal acceleration that changes the stellar geometry, its surface gravity, and the brightness distribution (Zorec et al. 2011). All these changes should be reflected in the differential phases of the spectral lines studied.

It is important to note that the gravity darkening law given by Eq. (1) is valid only when the proportionality between the local bolometric radiative flux and the effective gravity is constant over the stellar surface. As already noted in Sect. 1, this is possible only when 1) the rotation law in the stellar envelope is conservative (the angular velocity depends only on the distance to the rotation axis); 2) the envelope is in radiative equilibrium; and 3 ) the diffusion approximation to the radiative transfer holds.

The aim of this section is to provide an exploratory study of the effects shown in spectral lines by the GDE and the differential rotation when stars are seen at different inclination angles. As this is mainly an exploratory exercise, depending on the needs, we proceed with different degrees of approximation that are detailed according to the effect or the spectral line studied.

\subsection{The $\beta$ exponent}

The surface stellar brightness distributions are strongly dependent on the adopted darkening law. In the present discussion we write this law as follows:

$F_{\text {bol }}(\theta, \alpha)=c(\eta, \alpha) g_{\mathrm{eff}}^{4 \beta}(\theta, \alpha)$

where $F_{\text {bol }}(\theta, \alpha) \propto T_{\text {eff }}^{4}(\theta)$ is the local bolometric radiative flux and $T_{\text {eff }}(\theta)$ is the local effective temperature; $c(\eta, \alpha)$ is a function that should normally depend on both $\eta$ and $\alpha$, where $\alpha$ is the constant that parameterizes the degree of differential rotation, but in general possible also on $\theta$. Here, $\eta$ is the relation between the centrifugal acceleration at the equator $\left(F_{\mathrm{c}}\right)$ and the gravitational acceleration $\left(F_{\mathrm{g}}\right)$ at the equator, and $\alpha$ parameterizes the degree of surface differential rotation. We can assume that $c$ is still a constant dependent only on $\eta$, but the effect of $\alpha$ passed entirely on $\beta$, which accordingly becomes $\beta=\beta(\alpha, \beta, \theta)$. To put forward more clearly the effect of $\alpha$, we adopt $\beta$ to be constant and think of it as an average quantity over the co-latitude $\theta$. To assess the influence of $\alpha$ on the stellar brightness distribution, we changed the surface effective gravity adopting the Maunder relation for the surface angular velocity

$\Omega(\theta)=\Omega_{\mathrm{eq}}\left(1+\alpha \cos ^{2} \theta\right)$,

where $\Omega_{\mathrm{eq}}$ is the angular velocity at the equator. For $\alpha>0$ the angular velocity is accelerated toward the pole $(\partial \Omega / \partial \theta<0 ; \alpha$ can has any positive value), while for $\alpha<0$ angular velocity accelerates toward the equator ( $\partial \Omega / \partial \theta>0$; it must be $\alpha>-1.0$ to keep all stellar latitudes rotating in the same sense). The fit of the solar surface differential angular velocity $\Omega(\theta)$ law (Snodgrass 1984) with Eq. (5) produces $\alpha<-0.29$ (Zorec et al. 2011). The expression for the effective gravity $g_{\text {eff }}$ then becomes

$$
\left.\begin{array}{rl}
\frac{g_{\mathrm{eff}}(\theta)}{g_{\mathrm{p}}} & =\left(\frac{R_{\mathrm{p}}}{R_{\mathrm{eq}}}\right)^{2} \frac{1}{r^{2}(\theta)}\left\{1+\left\{\left[1-\eta_{\alpha}(\theta) r^{3}(\theta)\right]^{2}-1\right\} \sin ^{2} \theta\right\}^{1 / 2} \\
\eta_{\alpha}(\theta) & =\eta \times\left(1+\alpha \cos ^{2} \theta\right)^{2}
\end{array}\right\}
$$

with

$\eta=\frac{F_{\mathrm{c}}}{F_{\mathrm{g}}}=\frac{\Omega_{\mathrm{eq}}^{2} R_{\mathrm{eq}}^{3}}{G M_{\star}}=\left(\frac{\Omega_{\mathrm{eq}}}{\Omega_{\mathrm{c}}}\right)^{2}\left(\frac{R_{\mathrm{eq}}}{R_{\mathrm{c}}}\right)^{3}=\left(\frac{V_{\mathrm{eq}}}{V_{\mathrm{c}}}\right)^{2}\left(\frac{R_{\mathrm{eq}}}{R_{\mathrm{c}}}\right)$.

In Eq. (6), $r(\theta)$ is the radius-vector normalized to the equatorial radius $R_{\mathrm{eq}}$ that describes the geometry of the stellar surface. In Eq. (6), $g_{\mathrm{p}}$ is the polar gravity; $R_{\mathrm{p}}$ and $R_{\mathrm{c}}$ are the polar and equatorial critical radii, respectively; $M_{\star}$ is the stellar mass; $V_{\text {eq }}$ and $V_{\mathrm{c}}$ are the equatorial linear velocity at $\eta$ and critical velocity, respectively. The radius vector $r(\theta)$, radii $R_{\mathrm{p}}$ and $R_{\text {eq }}$ must be calculated consistently as a function of $\eta$ and $\alpha$ as indicated in Zorec et al. (2011). The effect in Eq. (6) of the centrifugal acceleration as a function of the co-latitude $\theta$ is produced by $\eta_{\alpha}(\theta)$ and $r(\theta)$.

To see the influence of $\alpha$ on the stellar brightness distribution, we computed several maps with different values of $\eta$ and $\alpha$. 

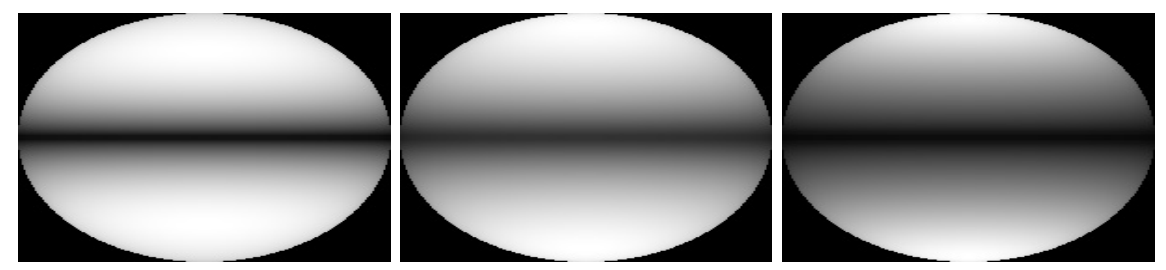

Fig. 9. Brightness distribution on the stellar disk in the continuum at $\lambda 4437 \AA$, near the conspicuous He I $4471 \AA$ A line, calculated for a star rotating at $\eta=0.9$ and for different values of $\alpha$ (from left to right): $-1.0,0.0$ and 1.5, seen at an inclination angle of $90^{\circ}$.

Table 4. Apparent $\beta_{\text {app }}$ exponents as a function of the $\alpha$ and $\eta$ parameters.

\begin{tabular}{lcccccc}
\hline \hline$\eta$ & 0.01 & 0.1 & 0.3 & 0.5 & 0.7 & 0.9 \\
$\Omega / \Omega_{\mathrm{c}}$ & 0.172 & 0.521 & 0.796 & 0.918 & 0.976 & 0.998 \\
$\alpha$ & & & & & & \\
\hline-1.0 & 0.250 & 0.253 & 0.261 & 0.267 & 0.271 & 0.274 \\
-0.5 & 0.250 & 0.252 & 0.256 & 0.260 & 0.262 & 0.263 \\
0.0 & 0.250 & 0.250 & 0.250 & 0.250 & 0.250 & 0.250 \\
0.5 & 0.250 & 0.247 & 0.242 & 0.239 & 0.236 & 0.234 \\
1.0 & 0.250 & 0.243 & 0.233 & 0.226 & 0.222 & 0.215 \\
1.5 & 0.250 & 0.239 & 0.223 & 0.214 & 0.208 & 0.204 \\
\hline
\end{tabular}

The maps obtained for $\lambda=4437 \AA, \eta=0.9, \beta=0.25$, and for an object characterized by $T_{\mathrm{p}}=19000 \mathrm{~K}, \log g_{\mathrm{p}}=4.0$ seen at $i=90^{\circ}$, and several values of $\alpha$ are shown in Fig. 9. In Fig. 9, it is clearly apparent that in rapid rotators, $\alpha$ has a strong effect on the surface brightness distribution. Thus, even with the value of $\beta=0.25$ (adopted for the calculations), the distribution of the surface brightness can be mis-interpreted in terms of apparent parameters, $\beta_{\text {app }} \neq \beta$, if the original von Zeipel relation is used to account for the GDE. The apparent $\beta_{\text {app }}$ can be estimated by dividing the stellar surface into elementary elements labeled with sub-indices $(i, j)$ that indicate the respective co-latitude and longitude angles $\left(\theta_{i}, \phi_{j}\right)$. Under the above adopted simplifications for the gravity darkening law, we let the parameter $\beta$ vary over the stellar surface and adopted an average estimate of it, which is given by $\beta_{\mathrm{app}}=\left\langle\beta_{i, j}\right\rangle$

$\beta_{\text {app }}=0.25 \times\left\{\frac{\sum_{i=1}^{n} \sum_{j=1}^{m}\left\{\ln g_{i, j}^{(0)}+\ln \left[F_{i, j}^{(\alpha)} / F_{i, j}^{(0)}\right]\right\}}{\sum_{i=1}^{n} \sum_{j=1}^{m} \ln g_{i, j}^{(\alpha)}}\right\}$.

where $g_{i, j}^{(0)}, g_{i, j}^{(\alpha)}$ are the effective gravities at $\left(\theta_{i}, \phi_{j}\right)$ for $\alpha=0$ and $\alpha \neq 0$, respectively; while $F_{i, j}^{(0)}, F_{i, j}^{(\alpha)}$ are the corresponding bolometric radiative fluxes. Table 4 summarizes the values of the apparent $\beta_{\text {app }}$ calculated for the stellar example presented in Fig. 9, except that calculations were made for several values of $\eta$

From Table 4 we can see that $\beta_{\text {app }} \gtrsim 0.25$ when $\alpha \lesssim 0.0$ and $\beta_{\text {app }} \lesssim 0.25$ if $\alpha \gtrsim 0.0$. The higher the value of $\eta$, the larger the differences between $\beta_{\text {app }}$ and $\beta$. These results show that the interpretation of $\beta_{\text {app }}$ deduced from observations deserves a thorough discussion before anything can be said regarding the law characterizing the stellar surface rotation, whether radiation or convection prevails on the stellar surface. All these calculations rely on the assumption that the stellar atmosphere is in radiative equilibrium and that the radiative flux responds to the temperature gradient as established by the diffusion approximation. Claret (2012) has shown, however, that this approximation may not hold. Under more detailed representations of the radiative flux we can also have a $\beta_{\text {app }} \lesssim 0.25$ effect that certainly relates in some way to the changes foreseen above as a function of $\alpha$ and $\beta$.

\subsection{The $\mathrm{H} \alpha$ line as a function of the inclination angle $i$ and differential rotation parameter $\alpha$}

Because we have at our disposal interferometric observations covering almost the entire $\mathrm{H} \alpha$ line of $\alpha$ Cep, in the present section, we explore whether we can use this line to estimate the inclination angle $i$ of the rotation axis. Knowing that the inclination angle is in principle a parameter of extrinsic character, it may be interesting to inquire on how much its determination may depend on the simultaneous knowledge of other intrinsic stellar quantities. A thorough discussion of this question is far beyond the scope of the present paper. We shall, however, briefly skim its dependence on the inclination angle $i$ and on the differential rotation parameter $\alpha$.

First, we calculated the $\mathrm{H} \alpha$ line emitted by the $i$-apparent stellar hemisphere by considering the stellar geometry controlled by a given $\eta$ and several values of the differential rotation parameter $\alpha$. Using the stellar geometry derived according to the rules given in Zorec et al. (2011), we assumed that each surface element is characterized by a plane-parallel stellar atmosphere corresponding to $\log g_{\text {eff }}(\theta)$, and $T_{\text {eff }}(\theta)$ derived from Eq. (4). All these requirements are taken into account in the calculation code FASTROT modified for surface differential rotation (Frémat et al. 2005; Zorec et al. 2011). Adopting the stellar fundamental parameters of $\alpha$ Cep obtained by Zhao et al. (2009), we calculated two series of $\mathrm{H} \alpha$ line profiles assuming rigid rotation, i.e., $\alpha=0.0$. In the first series, shown in Fig. 10, we adopted $V_{\mathrm{eq}}=267 \mathrm{~km} \mathrm{~s}^{-1} \approx[225 \pm 20] / \sin \left[55.7^{\circ} \pm 6.2^{\circ}\right]$ which implies $\eta \simeq 0.57$, and varied the inclination angle in the interval $35^{\circ}<i<90^{\circ}$. In this figure we clearly see the very low sensitivity of the $\mathrm{H} \alpha$ line profile to the $V \sin i$ parameter. In the second series, also shown in Fig. 10, we have adopted $V \sin i=225 \mathrm{~km} \mathrm{~s}^{-1}$ and varied the force-ratio in the interval $0.4<\eta<0.8$, which implies a change in the inclination angle from $46.7^{\circ}$ to $77.1^{\circ}$.

We see again that only the core of the line profile may show some significant changes as a function of $\eta$. For a third series of $\mathrm{H} \alpha$ line profiles we assumed $\eta=0.57$ for the rotation at the equator, so that $V \sin i=225 \mathrm{~km} \mathrm{~s}^{-1}$ suited for $i \simeq 56^{\circ}$ and adopted several values of the differential parameter in the interval $=-1.0<\alpha<+1.5$. The line profiles obtained are shown in Fig. 11, where we see that once a $V \sin i$ is adopted, the core of the line changes somewhat with $\alpha$.

In a second step, we calculated brightness distributions over the apparent stellar hemisphere in the wavelengths of the $\mathrm{H} \alpha$ line by considering that the stellar geometry is controlled by a given $\eta$ and differential rotation parameters $\alpha$, seen at various inclination angles. The respective differential phases as a function of the wavelength $\lambda$ inside the $\mathrm{H} \alpha$ line were calculated as before, with the physical parameters $T_{\mathrm{p}}, \log g_{\mathrm{p}}, \Omega / \Omega_{\mathrm{c}}, R_{\mathrm{eq}} / R_{\mathrm{p}}, R_{\mathrm{p}} / R_{\odot}$, and $\beta$ of $\alpha$ Cep found by Zhao et al. (2009) (see Table 1). We also considered the position angles or arrays during the observations carried out on $\alpha$ Cep from 2008 to 2010 and the corresponding baselines of VEGA/CHARA given in Table 2. For each baseline configuration and inclination angles $i$ ranging from $47.7^{\circ}$ to $75.7^{\circ}$ by a $3^{\circ}$ step, we obtained the differential phases shown in Fig. 12. If the method were sensitive enough 
O. Delaa et al.: Spectrally resolved interferometric observations of $\alpha$ Cep and physical modeling of fast rotating stars
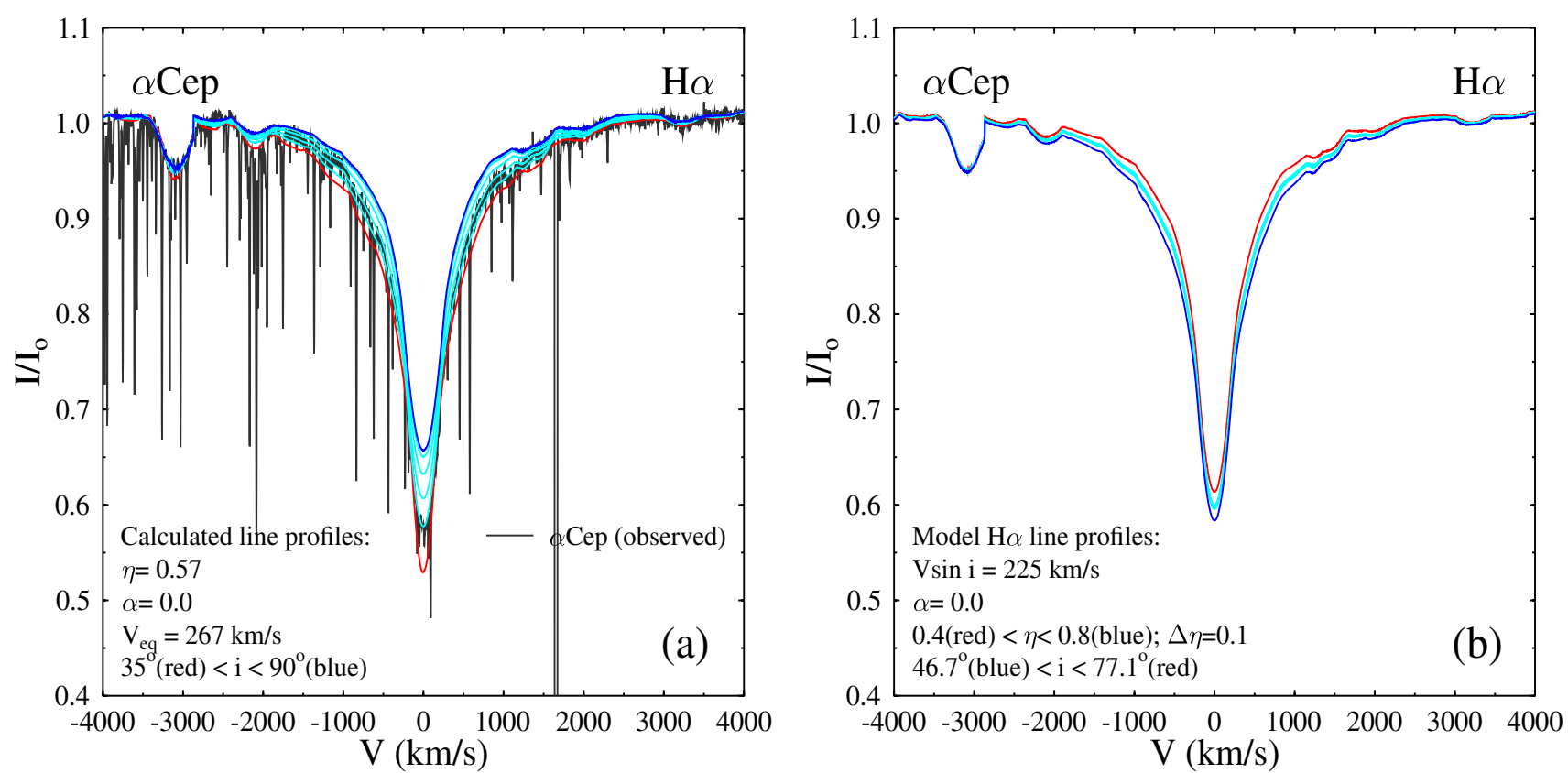

Fig. 10. Rotationally broadened $\mathrm{H}_{\alpha}$ line profiles using the fundamental parameters of $\alpha$ Cep and assuming rigid rotation. a) Line profiles adopting $\eta=0.57$ (or $V_{\text {eq }}=267 \mathrm{~km} \mathrm{~s}^{-1}$ ) and varying the inclination angle. For comparison sake, we have included the observed $\mathrm{H}_{\alpha}$ line profile of $\alpha$ Cep. b) Line profiles adopting $V_{\mathrm{eq}} \sin i=225 \mathrm{~km} \mathrm{~s}^{-1}$ and varying $\eta$ (or the inclination angle $i$ ).

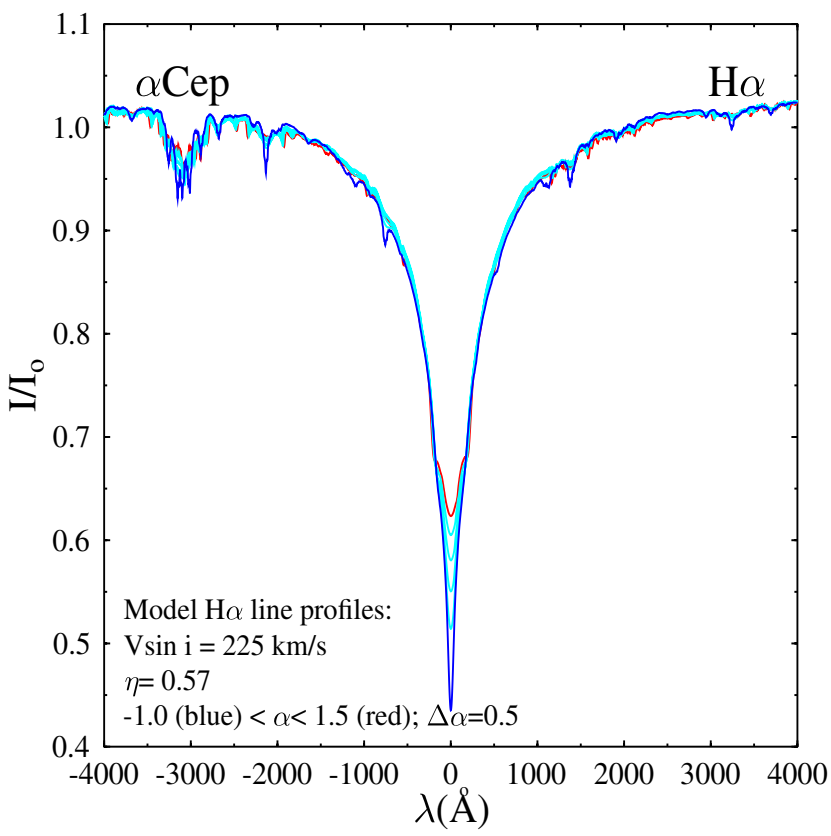

Fig. 11. Rotationally broadened $\mathrm{H}_{\alpha}$ line profiles using the fundamental parameters of $\alpha$ Cep and surface differential rotation. The line profiles are plotted for $\eta=0.57, V \sin i=225 \mathrm{~km} \mathrm{~s}^{-1}$ and for several values of $\alpha$ of the Maunder relation for surface differential rotation.

to the inclination $i$, the interval chosen for the tested inclination angle $i$ would, in principle, enable us to distinguish between the estimates of $i$ by van Belle et al. (2006) and Zhao et al. (2009). The respective largest amplitudes of deviations of $\phi_{\text {diff }}(\lambda)$ obtained are given in Table 5, which show that for the configurations of the interferometric array used, our observations of the $\mathrm{H} \alpha$ line can hardly lead to conclusive results.

In spite of these results, we repeated the calculation of differential phases for a CHARA Array optimal configuration,
Table 5. Strongest variation of $\phi_{\text {diff }}(\lambda)$ across the $\mathrm{H} \alpha$ line of $\alpha$ Cep as a function inclination angles $i$ ranging from $47.7^{\circ}$ to $i=-75.7^{\circ}$.

\begin{tabular}{lc}
\hline \hline Array & $\begin{array}{c}\text { Strongest variation of } \\
\phi_{\text {diff }}(\lambda)\end{array}$ \\
\hline $\mathrm{S} 1 \mathrm{~S} 2_{a}$ & $0.84^{\circ}$ \\
$\mathrm{S} 1 \mathrm{~S} 2_{b}$ & $0.58^{\circ}$ \\
$\mathrm{S} 1 \mathrm{~S} 2_{c}$ & $0.11^{\circ}$ \\
$\mathrm{E} 1 \mathrm{E} 2_{a}$ & $2.55^{\circ}$ \\
$\mathrm{E} 1 \mathrm{E} 2_{b}$ & $1.43^{\circ}$ \\
$\mathrm{E} 1 \mathrm{E} 2_{c}$ & $2.20^{\circ}$ \\
\hline
\end{tabular}

represented by a baseline of 63 meters and position angle PA = $157^{\circ}$. Using the same stellar physical parameters and inclination angles $0^{\circ} \leq i \leq 90^{\circ}$ as before, we obtained the differential phases shown in Fig. 13. In the left panel are shown differential phases when $V \sin i=225 \mathrm{~km} \mathrm{~s}^{-1}$ is conserved and the inclination angle varied from in the interval $35^{\circ}<i<90^{\circ}$. We can see in this figure that the maximum amplitude of the phase variation is slightly larger than the uncertainty on the observational differential phase estimate $\left(\sigma_{\phi} \simeq 3^{\circ}\right)$. In the left panel, models keep $V\left(=267 \mathrm{~km} \mathrm{~s}^{-1}\right)$ constant but vary the inclination angle from $i=0^{\circ}$ to $i=90^{\circ}$. Since this is another diagnostic way to interpret the observed differential phases, we see that the method leads to amplitude changes of phases that are larger than $\Delta \phi=5 \times \sigma_{\phi}$. We also note that changes are also seen in the position of maxima larger than $10 \mathrm{~km} \mathrm{~s}^{-1}$, which provide a supplementary tool for interpreting the observations. We may then expect that observations carried on the $\mathrm{H} \alpha$ line might lead us to some estimate of the inclination angle $i$ if they are performed at optimal conditions, which unfortunately were not fulfilled in the present case. Finally, we note that model calculations should be made with fundamental parameters corresponding to the parent, non-rotating object. This implies that the estimate of $i, \alpha$, and of the remaining stellar fundamental parameters have to be iterated. These problems will be discussed in greater detail in a forthcoming paper. 

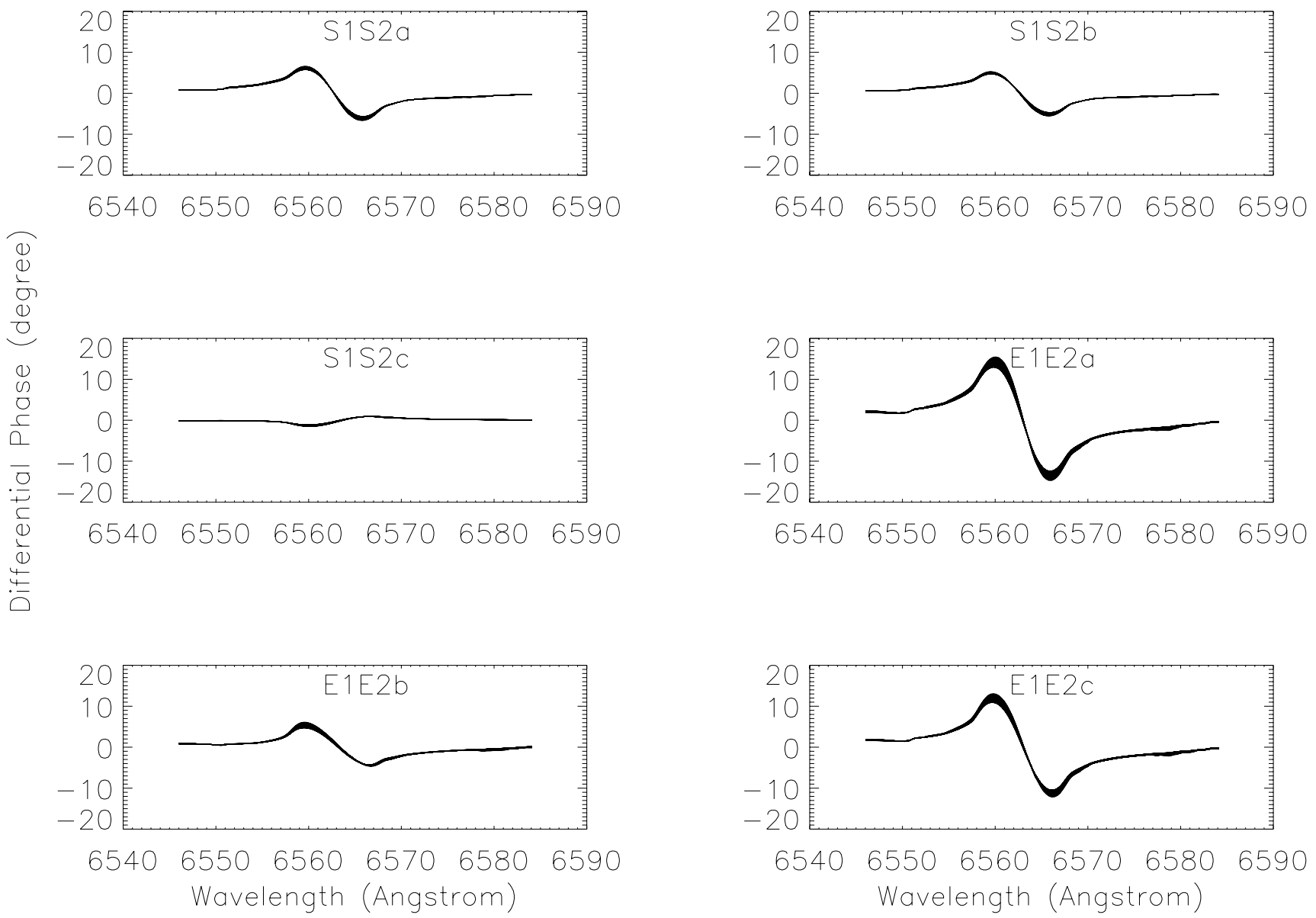

Fig. 12. Estimated variations of the differential phases as function of the inclination (from $47.7^{\circ}$ to $75.7^{\circ}$ ) of $\alpha$ Cep across the $\mathrm{H}_{\alpha}$ line for our six observations. Calculations were performed with $\mathrm{PA}=-158^{\circ}$.

\subsection{Properties of spectral lines and the determination of $i$ and $\alpha$}

To understand better the lack of results on $i$ and $\alpha$ obtained for $\alpha$ Cep from studies based on the $\mathrm{H} \alpha$ line, we considered the rotational broadening and differential phases as generalized convolutions between the line flux profile and a rotational broadening-like (RB) function. To this end, we note that the width of the rotational broadening function proper is about $\Delta \lambda_{\mathrm{RB}}=\lambda_{\mathrm{H} \alpha} V \sin i / c=5.6 \AA$, while the $\mathrm{H} \alpha$ line spreads over some $\Delta \lambda_{\mathrm{H} \alpha} \simeq 80 \AA$. This means that the several intervening rotational broadening functions used should also behave like quasidelta functions that nearly preserve the shape of the original spectral line profile, except perhaps in the line core wavelengths. Because the broad width of the $\mathrm{H} \alpha$ line is due to the Stark effect, to obtain more detailed information on the rotational broadening, in particular that produced by a surface differential rotation, spectral lines as free as possible from Stark broadening should be employed.The $\mathrm{H} \alpha$ line wings are sensitive to $\log g$, so a variation of the line profile in rapidly rotating stars induced by the $\theta$-dependent $\log g_{\text {eff }}$ is expected. Nevertheless, the rapidly rotating equatorial regions where both $\log g_{\text {eff }}$ and the effective temperature are low do not contribute efficiently to the global line flux. The Doppler broadening of the line is thus left to the higher stellar latitudes where $\log g_{\text {eff }}$ is comparatively larger and more uniform.

Finally, the nature of the $\mathrm{H} \alpha$ line source function also deserves some attention. In stars with $T_{\text {eff }} \gtrsim 8000 \mathrm{~K}$, the $\mathrm{H} \alpha$-source function is dominated by photo-ionizations, which make the line quite non-local in character (Thomas 1965, 1983; Mihalas 1978). Consequently, the $\mathrm{H} \alpha$ line formation is fairly independent of details concerning the non-uniform distributions of $T_{\text {eff }}(\theta)$ and $\log g_{\text {eff }}(\theta)$ induced by the GDE. Because we expect that the surface differential rotation is correlated with physical condition changes in the line formation regions, to increase the degree of information on the external rotation law of stars, we should use spectral lines that not only carry signatures of the macroscopic broadening velocity fields, but also have source functions sensitive to changes of local physical conditions $\left(T_{\mathrm{e}}, N_{\mathrm{e}}\right)$. This means that these spectral lines should have source functions that are collision-dominated.

\subsection{Information expected from spectral lines sensitive to effects induced by the rotation}

According to spectral type and luminosity class, several spectral lines can be good candidates to carry out such studies. Since the identification of these lines and the subsequent calculation of expected effects from all of them is far beyond the scope of the present paper, we limit our discussion to a hypothetical line that behaves like the $\mathrm{He} I \lambda 4471$ line, which fulfills the above quoted requirements in stars with $T_{\text {eff }} \gtrsim 10000$. Though this line is not useful for $\alpha$ Cep, our aim is to briefly sketch some expected effects of quite general character.

Because the He I $\lambda 4471$ line is only a hypothetical model line, we do not proceed with detailed radiation transfer 
O. Delaa et al.: Spectrally resolved interferometric observations of $\alpha$ Cep and physical modeling of fast rotating stars
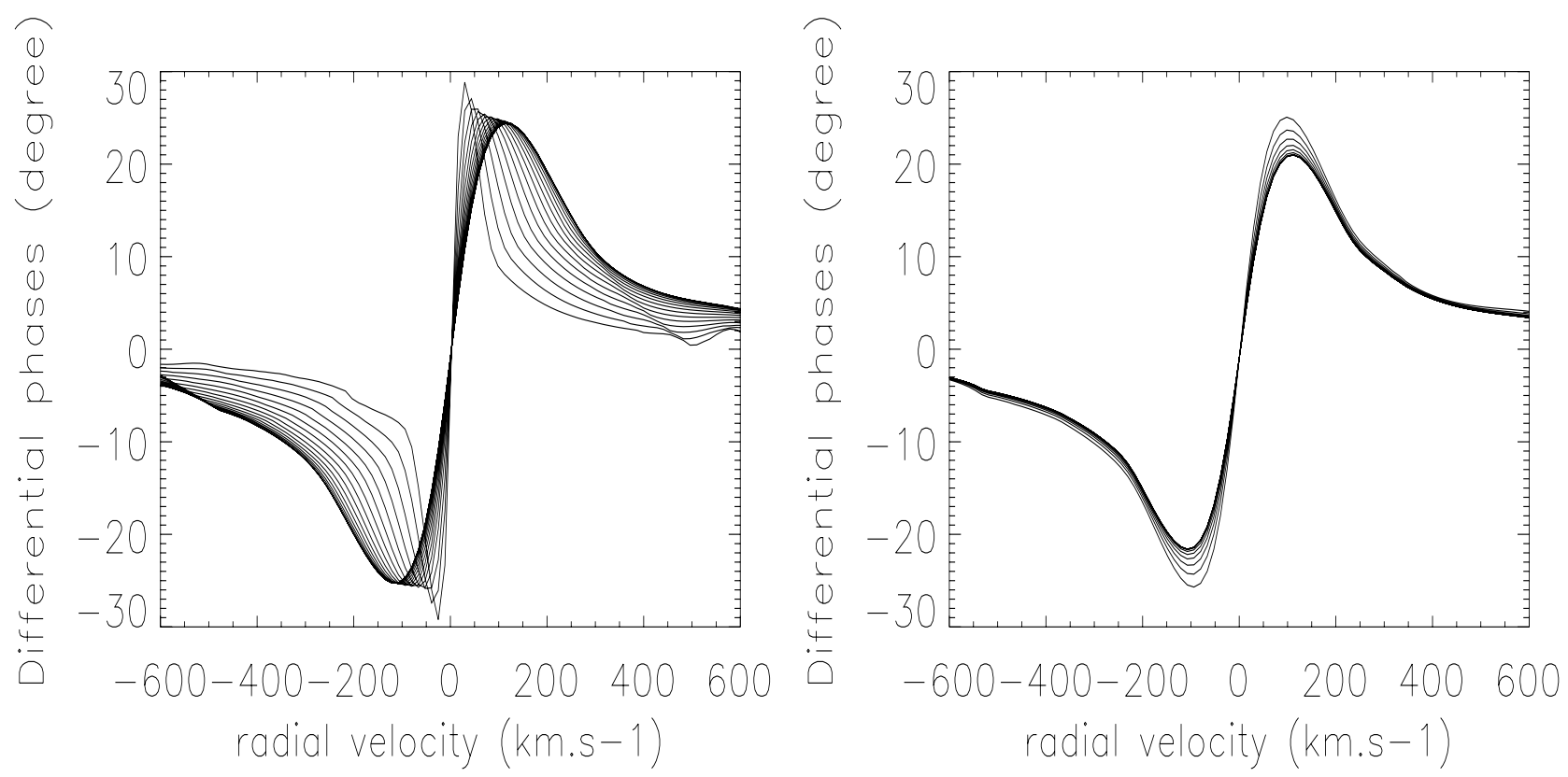

Fig. 13. Differential phases $\phi_{\text {diff }}(\lambda)$ over the $\mathrm{H} \alpha$ line for different inclination angles $i$ of $\alpha$ Cep. Computations were done for a baseline of $63 \mathrm{~m}$ and baseline orientation $-158^{\circ}$. Left panel: differential phases for $V \sin i=225 \mathrm{~km} \mathrm{~s}^{-1}$ fixed, but varying inclination angle from $i=35^{\circ}$ to $i=90^{\circ}$ by steps $\Delta i=5^{\circ}$ and $\eta$ accordingly. Right panel: differential phases for $V=267 \mathrm{~km} \mathrm{~s}^{-1}$ fixed $(\eta=0.57)$ and inclination angles varying from $i=10^{\circ}$ to $i=90^{\circ}$ by steps $\Delta i=5^{\circ}$. The parent star without rotation has $T_{\text {eff }}=7500 \mathrm{~K}$ and $\log g=4.0$.

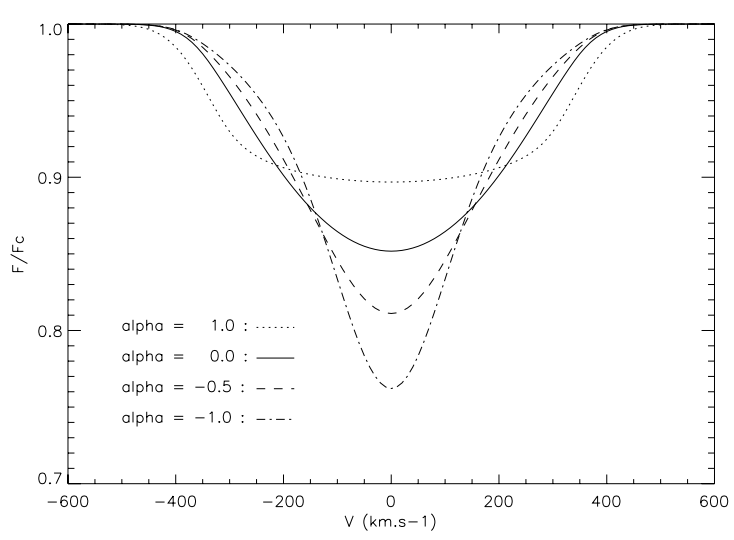

Fig. 14. He I 4471-like line profiles as a function of different values of the differential rotation parameter $\alpha$. The star is characterized by $T_{\mathrm{p}}=$ $19000 \mathrm{~K}, \log g_{\mathrm{p}}=4.0, \eta=0.9, \beta=0.25$ and $i=45^{\circ}$.

calculations, but represent the emergent monochromatic specific intensity $I(\lambda, \mu)$ directed toward the observer as follows:

$I(\theta, \phi, \eta, \alpha, \lambda, \mu)=H\left[\theta, \lambda-\lambda_{o} \frac{V_{\text {proj }}(\theta, \phi, \alpha, i)}{c}\right] I_{\mathrm{c}}(\theta, \eta, \alpha, \mu)$,

where $\theta$ reflects the dependence of $I(\lambda, \mu)$ with the local $T_{\text {eff }}(\theta)$ and $\log g_{\text {eff }}(\theta)$, whose variation with $\theta$ is determined by $\eta$ and $\alpha$; the dependence of $I(\lambda, \mu)$ with the longitude $\phi$ is due to the law of the surface differential rotation that produces iso-radial velocity curves that are a function of $\theta$ and $\phi$ over the apparent stellar hemisphere; $\eta$ controls the equatorial rotational velocity, and $\alpha$ parameterizes the surface differential rotation according to the Maunder law; $I_{\mathrm{c}}(\theta, \eta, \alpha, \mu)$ is the specific radiation intensity directed toward the observer in the continuum at the central wavelength $\lambda_{\mathrm{c}}$ of the studied line; $\mu(\theta, \phi, i)=\cos (\hat{n}, \hat{\beta})=$ $\hat{n} . \hat{\beta}$, where $\hat{n}=\bar{g}_{\text {eff }}(\theta) /\left|g_{\text {eff }}(\theta)\right|$ and $\hat{\beta}=\cos i \hat{e}_{y}+\sin i \hat{e}_{z}$ are the

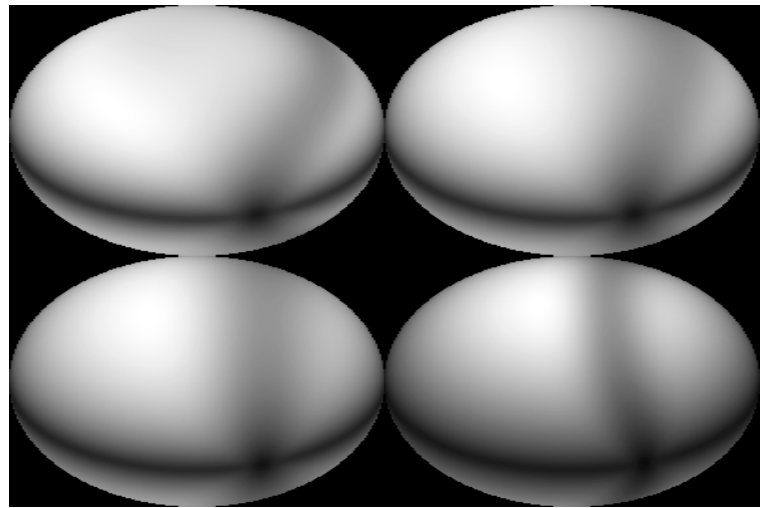

Fig. 15. Iso-radial velocity zone for $\lambda=4438.78 \AA$. Top, form left to right: $\alpha=-1.0$ and -0.5 . Bottom, from left to right: $\alpha=0.0$ and 1.0 . computations were done for an inclination angle of $i=45^{\circ}$.

unit normal vector to the stellar surface and the unit vector directed toward the observer, respectively. The specific intensity $I_{\mathrm{c}}$ is represented as

$I_{\mathrm{c}}(\theta, \eta, \alpha, \mu)=\frac{F_{\mathrm{c}}(\theta)}{\pi\left(1-\epsilon_{\lambda_{\mathrm{c}}} / 3\right)}\left(1-\epsilon_{\lambda_{\mathrm{c}}}+\epsilon_{\lambda_{\mathrm{c}}} \cos \mu\right)$,

where $F_{\mathrm{c}}(\theta)$ is the emergent continuum radiation flux $\lambda_{\mathrm{c}}$ at $\lambda_{\mathrm{c}}$; $\epsilon_{\lambda_{\mathrm{c}}}$ is the limb darkening coefficient $\epsilon_{\lambda}$ at $\lambda_{\mathrm{c}}$ of the continuum spectrum that we interpolated in Claret (2012) for the solar chemical abundance and the local $\left[T_{\text {eff }}(\theta), \log g_{\text {eff }}(\theta)\right]$. In a more detailed treatment of spectral lines, the limb darkening coefficient should vary with the wavelength inside the spectral line (Collins \& Truax 1995; Chauville et al. 2001).

Let $(x, y, z)$ be a Cartesian reference system centered on the apparent stellar disk, where $(x, y)$ are in the plane of the sky, $z$ is directed towards the observer, $y$ is in the plane containing $z$ and 
A\&A 555, A100 (2013)
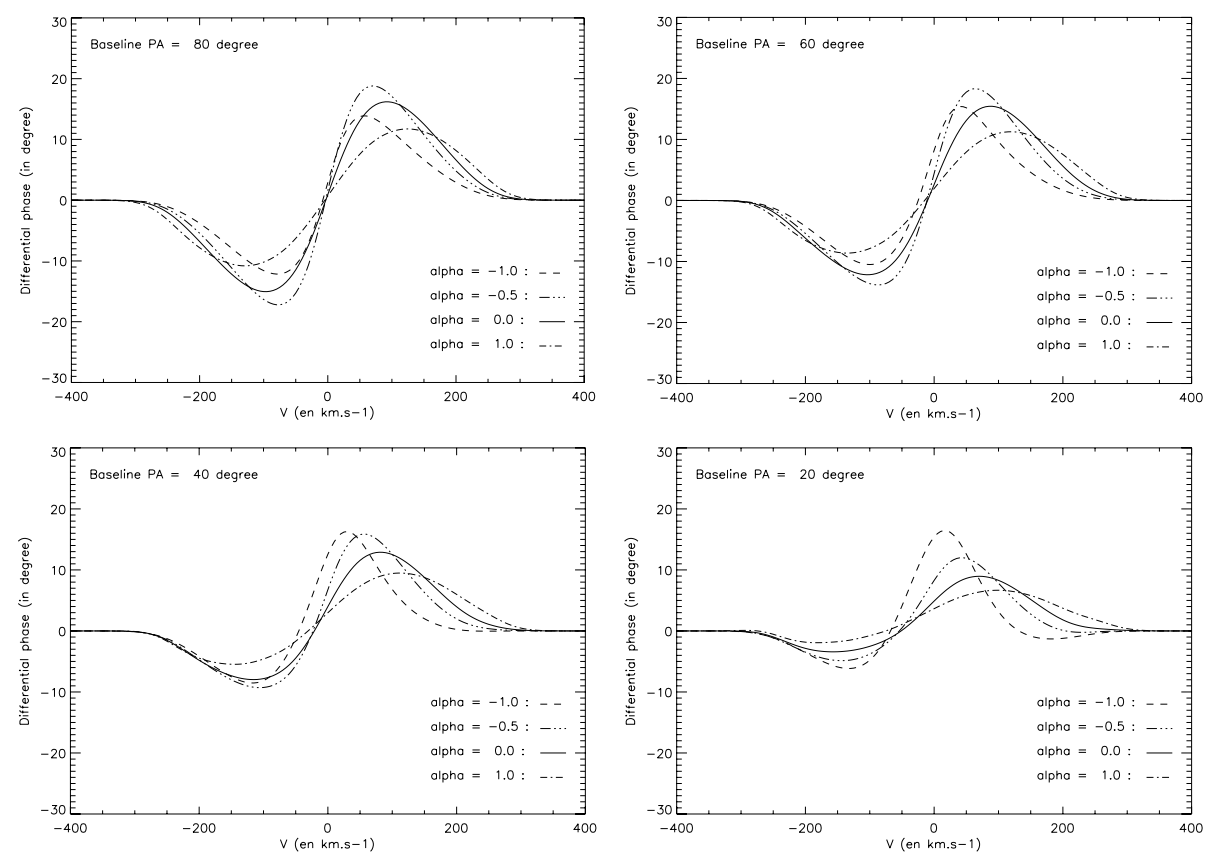

Fig. 16. Evolution of the differential phase as a function of the wavelength through the He I line for different values of $\alpha$. Differential phases were computed for different orientations and a $60-\mathrm{m}$ baseline. The position angle of the star is assumed to be equal to zero, the inclination angle of the rotation axis is $i=45^{\circ}$, and the stellar angular diameter is assumed to be 1.3 mas. the stellar rotation axis. The radial velocity projected towards the observer of a surface point $(r, \theta, \phi)$ is then

$$
\left.\begin{array}{rl}
V_{\text {proj }}(\theta, \phi, \eta, \alpha, i) & =[\overline{\Omega(\theta)} \wedge \overline{\boldsymbol{r}(\theta, \eta, \alpha)}] . \hat{\beta} \\
& =\Omega(\theta, \alpha) R_{\mathrm{eq}}(\eta) x(\eta, \theta, \phi) \sin i \\
x(\eta, \theta, \phi) & =\boldsymbol{r}(\theta, \eta, \alpha) \sin \theta \sin \phi,
\end{array}\right\}
$$

where $\Omega(\theta, \alpha)$ is given by Eq. (5); $i$ is the inclination angle of the stellar rotation axis; $\boldsymbol{r}(\theta, \eta, \alpha)$ is the radius vector a point in the stellar surface.

The calculation of the flux line profile $F(\lambda)$ was made as in all preceding calculations: the star was divided into surface elements to which were associated with the respective $T_{\text {eff }}(\theta)$, $\log g_{\text {eff }}(\theta)$ and $V_{\text {proj }}(\theta, \phi, \alpha, i)$ that enable one to obtain the local Doppler-shifted specific intensity directed toward the observer. The integration over the visible stellar hemisphere of the projected specific intensities produces $F(\lambda)$. The central intensity and equivalent widths dependent on $\left[T_{\text {eff }}, \log g_{\text {eff }}\right]$ needed to determine the intrinsic line profile $H(\lambda)$ lower case In Eq. (8) were taken from Frémat et al. (2005). Equation (9) was used to obtain the local specific radiation intensity, either monochromatic or bolometric, directed toward the observer, which determines the sought distribution of the surface brightness. For the test examples calculated here, the input parameters were the polar effective temperature $T_{\mathrm{p}}=19000 \mathrm{~K}$, the polar gravity $g_{\mathrm{p}}=4.0$, the inclination angle $i=45^{\circ}$, gravity darkening exponent of the gravity darkening law $\beta=0.25$, the ratio $\eta=0.9$, and several values of the differential rotation parameter $\alpha$. The test He I $\lambda 4471$ line-like profiles obtained are shown in Fig. 14, where the strong variation of the line profiles according to the value of $\alpha$ is clearly apparent.

The corresponding maps of brightness distribution containing the iso-radial velocity zone for $\lambda=4439 \AA$, calculated for the same fundamental parameters, are shown in Fig. 15. At the top of this figure, from left to right, the images are for $\alpha=-1.0$ and -0.5 , while in the bottom panels we have from left to right, $\alpha=0.0$ and 1.0. We can clearly see in this figure that the regions crossed by a given iso-radial velocity curve are quite different. The iso-radial velocity curves thus run across zones with different $\left(T_{\text {eff }}, \log g\right)$ contributing to the final line flux profile with local line profiles having different intensities and Doppler broadenings. In Fig. 16 we note that according to the parameter $\alpha$, not only the amplitude variations of differential phase are stronger than the expected uncertainties affecting the observed differential phases, but the strongest phase deviations are radial-velocity shifted, which are $\alpha$-dependent. In this figure we notice that deviations of maxima in the differential phases exceed $10 \mathrm{~km} \mathrm{~s}^{-1}$ and the amplitude variation of this maximum widely exceeds $3^{\circ}$. These limits correspond to the VEGA/CHARA sensitivity. A spectral line in $\alpha$ Cep that would behave like the witness spectral line treated here could lead us to reliable inferences of the differential parameter $\alpha$. Looking for appropriate configurations of the interferometric array might improve the estimates even more.

\section{Conclusions}

Differential phases in the $\mathrm{H} \alpha$ line of $\alpha$ Cep observed during six observations at the VEGA/CHARA array in September 2008 and October 2010 were presented. We successfully applied a method for determining the position angle of the rotationally deformed star that takes advantage of the differential phases obtained at different configurations of the interferometric array, and is independent of the knowledge of the stellar fundamental parameters. We have thus obtained $\mathrm{PA}=-157_{-10^{\circ}}^{\circ}$.

We discussed the feasibility of determining differential phases using the gravity darkening exponent $\beta$ in von Zeipel's relation, the inclination angle $i$ of the star, and the differential rotation parameter $\alpha$ of Maunders's expression for the stellar surface rotation law. The discussion showed that the exponent $\beta$ is a function of the rotation rate $\eta=F_{\mathrm{c}} / F_{\mathrm{g}}\left(F_{\mathrm{c}}=\right.$ centrifugal acceleration at the equator; $F_{\mathrm{g}}=$ gravitational acceleration) and of $\alpha$. Its determination is probably not straightforward and needs a careful discussion of interferometric imaging of stellar surfaces.

From models of the $\mathrm{H} \alpha$ emitted by a rapidly rotating star with the fundamental parameters $\alpha \mathrm{Cep}$, we showed that for very ideal configurations of interferometric arrays it would be possible to estimate the inclination angle $i$. Nevertheless, a knowledge 
of the stellar fundamental parameters is needed. They can be inferred by iteration together with the inclination angle $i$. We finally concluded that spectral lines that are not strongly affected by Stark broadening and have collision-dominated source functions should be observed to estimate $i$ and $\alpha$ with differential phases. They are sensitive to these quantities in three different ways: height of the deviation wings, the value of the maximum deviation, and the spectral position of the maximum deviation.

Acknowledgements. VEGA is a collaboration between CHARA and OCA/LAOG/CRAL/LESIA that has been supported by the French programs PNPS and ASHRA, by INSU and by the Région PACA. The project has obviously benefitted from the strong support of the OCA and CHARA technical teams. The CHARA Array is operated with support from the National Science Foundation through grant AST-0908253, the W. M. Keck Foundation, the NASA Exoplanet Science Institute, and from Georgia State University. This work has made use of the BeSS database, operated at GEPI, Observatoire de Meudon, France: http://basebe.obspm. fr, use of the Jean-Marie Mariotti Center SearchCal service ${ }^{1}$ co-developed by FIZEAU and LAOG, and of CDS Astronomical Databases SIMBAD and VIZIER ${ }^{2}$. We are grateful to an anonymous referee for her/his valuable suggestions that helped to improve the presentation of our results.

\section{References}

Abt, H. A., \& Morrell, N. I. 1995, ApJS, 99, 135

Aufdenberg, J. P., Mérand, A., Coudé du Foresto, V., et al. 2006, ApJ, 645, 664

Berio, P., Mourard, D., Bonneau, D., et al. 1999, J. Opt. Soc. Am. A, 16, 872

Bernacca, P. L., \& Perinotto, M. 1973a, in Main sequence single stars, A catalogue of stellar rotational velocities (Universita di Padova), 1

Bernacca, P. L., \& Perinotto, M. 1973b, in Main sequence spectroscopic binaries, A catalogue of stellar rotational velocities (Universita di Padova), 2

Bernacca, P. L., \& Perinotto, M. 1973c, in Evolved stars, A catalogue of stellar rotational velocities (Universita di Padova), 4

Bowman, A. W., \& Azzalini, A. 1997, Applied smoothing techniques for data analysis: the kernel approach with S-plus illustrations, Oxford statistical science series No. 18 (Clarendon Press)

Chauville, J., Zorec, J., Ballereau, D., et al. 2001, A\&A, 378, 861

Che, X., Monnier, J. D., Zhao, M., et al. 2011, ApJ, 732, 68

Claret, A. 2012, A\&A, 538, A3

Collins, II, G. W., \& Truax, R. J. 1995, ApJ, 439, 860

Domiciano de Souza, A., Vakili, F., Jankov, S., Janot-Pacheco, E., \& Abe, L. 2002, A\&A, 393, 345

Domiciano de Souza, A., Kervella, P., Jankov, S., et al. 2003, A\&A, 407, L47

Domiciano de Souza, A., Hadjara, M., Vakili, F., et al. 2012a, A\&A, 545, A130

Domiciano de Souza, A., Zorec, J., \& Vakili, F. 2012b, SF2A Proc., in press

Douglas, A. V. 1926, ApJ, 64, 262

Efron, B., \& Tibshirani, R. 1986, Statist. Sci., 1, 54

Endal, A. S., \& Sofia, S. 1979, ApJ, 232, 531
Espinosa Lara, F., \& Rieutord, M. 2012, A\&A, 547, A32

Frémat, Y., Zorec, J., Hubert, A.-M., \& Floquet, M. 2005, A\&A, 440, 305

Gray, R. O., Corbally, C. J., Garrison, R. F., McFadden, M. T., \& Robinson, P. E. 2003, AJ, 126, 2048

Heger, A., \& Langer, N. 2000, ApJ, 544, 1016

Hubeny, I., \& Lanz, T. 1995, ApJ, 439, 875

Johnson, H. L., \& Morgan, W. W. 1953, ApJ, 117, 313

Kervella, P., \& Domiciano de Souza, A. 2006, A\&A, 453, 1059

Kiziloglu, N., \& Civelek, R. 1996, A\&A, 307, 88

Kurucz, R. L. 1979, ApJS, 40, 1

Lamers, H. J. G. L. M., \& Cassinelli, J. P. 1999, Introduction to Stellar Winds (Cambridge University Press)

Le Bouquin, J.-B., Absil, O., Benisty, M., et al. 2009, A\&A, 498, L41

Lucy, L. B. 1967, ZAp, 65, 89

Maeder, A., Meynet, G., \& Ekström, S. 2007, in From Stars to Galaxies: Building the Pieces to Build Up the Universe, eds. A. Vallenari, R. Tantalo, L. Portinari, \& A. Moretti, ASP Conf. Ser., 374, 13

Maeder, A., Georgy, C., \& Meynet, G. 2008, A\&A, 479, L37

McAlister, H. A., ten Brummelaar, T. A., Gies, D. R., et al. 2005, ApJ, 628, 439

Meynet, G., \& Maeder, A. 2000, A\&A, 361, 101

Mihalas, D. 1978, Stellar atmospheres, 2nd Ed. (San Francisco: W. H. Freeman and Co.)

Monnier, J. D., Zhao, M., Pedretti, E., et al. 2007, Science, 317, 342

Mourard, D. 1990, Ph.D. Thesis, Université de Nice

Mourard, D., Clausse, J. M., Marcotto, A., et al. 2009, A\&A, 508, 1073

Mourard, D., Tallon, M., Bério, P., et al. 2010, in SPIE Conf. Ser., 7734

Pinsonneault, M. H., Deliyannis, C. P., \& Demarque, P. 1991, ApJ, 367, 239

Reiners, A. 2003, A\&A, 408, 707

Royer, F., Zorec, J., \& Gómez, A. E. 2007, A\&A, 463, 671

Sackmann, I. J. 1970, A\&A, 8, 76

Shimazaki, H., \& Shinomoto, S. 2007, Neural Computation, 19, 1503

Simon, T., \& Landsman, W. B. 1997, ApJ, 483, 435

Simon, T., Ayres, T. R., Redfield, S., \& Linsky, J. L. 2002, ApJ, 579, 800

Snodgrass, H. B. 1984, Sol. Phys., 94, 13

Talon, S., Zahn, J.-P., Maeder, A., \& Meynet, G. 1997, A\&A, 322, 209

Tatulli, E., Millour, F., Chelli, A., et al. 2007, A\&A, 464, 29

ten Brummelaar, T. A., McAlister, H. A., Ridgway, S. T., et al. 2005, ApJ, 628 453

Thomas, R. N. 1965, Some aspects of non-equilibrium thermodynamics in the presence of a radiation field (University of Colorado Press)

Thomas, R. N. 1983, Stellar atmospheric structural patterns, 471 (NASA-CNRS)

Townsend, R. H. D. 1997, MNRAS, 284, 839

Tuominen, I. V. 1972, Ann. Acad. Sci. Fennicae, 392

Uesugi, A., \& Fukuda, I. 1970, Catalogue of rotational velocities of the stars (University of Kyoto)

van Belle, G. T., Ciardi, D. R., Thompson, R. R., Akeson, R. L., \& Lada, E. A. 2001, ApJ, 559, 1155

van Belle, G. T., Ciardi, D. R., ten Brummelaar, T., et al. 2006, ApJ, 637, 494

von Zeipel, H. 1924, MNRAS, 84, 665

Walter, F. M., Matthews, L. D., \& Linsky, J. L. 1995, ApJ, 447, 353

Zahn, J.-P. 1992, A\&A, 265, 115

Zhao, M., Monnier, J. D., Pedretti, E., et al. 2009, ApJ, 701, 209

Zorec, J., Frémat, Y., Domiciano de Souza, A., et al. 2011, A\&A, 526, A87

\footnotetext{
1 Available at http://www . jmmc. fr/searchcal

2 Available at http://cdsweb.u-strasbg.fr/
} 